\title{
A hybrid method for linearized wave radiation and diffraction problem by a three dimensional floating structure in a polynya
}

\author{
Z. F. $\mathrm{Li}^{\mathrm{a}}, \mathrm{Y} . \mathrm{Y} . \mathrm{Shi}^{\mathrm{b}}$ and G. X. Wu ${ }^{\mathrm{c}}{ }^{*}$
}

${ }^{a}$ School of Naval Architecture and Ocean Engineering, Jiangsu University of Science and Technology, Zhenjiang 212003, China

${ }^{\mathrm{b}}$ College of Shipbuilding Engineering, Harbin Engineering University, Harbin 150001, China

${ }^{\mathrm{c}}$ Department of Mechanical Engineering, University College London, Torrington Place, London WC1E 7JE, UK

\section{Abstract}

A hybrid method is developed to solve the interaction problem of wave with a three dimensional floating structure in a polynya. The linearized velocity potential theory is used for fluid flow, and the thin elastic plate model is adopted for the infinitely extended ice sheet. Because of sudden change of the upper boundary of the computational domain, namely from the ice sheet to the free surface, the domain is divided into two sub-domains, one below free surface and the other below the ice sheet. The solution method is divided into three components. The first component is the integral equation over the structure surface and the interface of the two sub-domains. In the second component, the velocity potential is expanded into a series of eigenfunctions in the vertical directions, which avoids the numerical difficulty in calculation of the fifth derivatives. This is coupled with a series of integral equations along the edge of the ice sheet. In the third component of the method, two orthogonal inner products are used to impose the continuity conditions of the velocity and pressure on the interface, as well as the boundary conditions on the ice edge. The developed method is verified through comparison with the analytical solution for a circular cylinder. Case study is then made for a FPSO in a polynya with different shapes and floating positions. The hydrodynamic coefficients, wave exciting force and wave elevation in polynya are provided and analyzed.

Keywords: hybrid method; polynya; flexural-gravity wave; free surface wave; hydrodynamic load

\section{Introduction}

Accurate prediction of wave-induced loads is very important in both the design and operation of offshore structures. Motivated by the new potentials in natural resources, there is major interest in building structures for the far north Arctic regions, such as drilling rigs, oil-gas production facilities [1]. This has led to some new challenges to the hydrodynamics, as the platform may

\footnotetext{
* Corresponding author. E-mail address: g.wu@ucl.ac.uk (G.X. Wu).
} 
operate in different icy water conditions, e.g. near large icebergs or ice blocks, floating in a polynya surrounded by large ice sheet. The last case will be considered in this work and solved by an efficient hybrid method based on the velocity potential theory.

There has been extensive research over the many decades on the free surface wave interactions with a structure in open sea, and various numerical methods have been developed (e.g. [2-4]). These methods are nowadays well established and their applications have significantly advanced our understanding of water wave interactions with structures. Among them is the boundary element method which transforms the governing Laplace equation for the velocity potential into the boundary integral equation over the boundary, with the help of a Green function. In general, the integral equation is over the entire boundary. For the linearized problem, however, the Green function satisfying all the boundary conditions, apart from that on the structure surface can be derived. In such a case, the integral equation involves only the body surface and the need to discretize the free surface is removed. The method has then been widely used in the free surface problem (e.g. [5-7]). In [7], the integral was over an vertical circular cylindrical surface and performed analytically, which was then used as a radiation condition for the inner domain with Rankine source as the Green function.

For the problem considered in the present paper, a body floating on the free surface of a polynya surrounded by ice sheet, it is not straight forward to derive the Green function satisfying both the free surface boundary conditions in the polynya and the ice sheet boundary conditions. An alternative may be to use the so called Rankine source method. In such a case, both the ice sheet and free surface have to be discretized, together with the body surface. It will increase the number of panels drastically. More importantly, there are higher derivatives, up to fifth one, on the ice sheet (see [8-10] for example). To deal with higher derivatives in the integral equation is always difficult because of the singular behaviour of the Green function. In fact, even for the second derivative, some special method is needed (e.g. $[11,12])$. Thus for the two dimensional problem (2D), a hybrid method was introduced [13]. In the polynya, the boundary element method was used. In the region below the ice sheet, the potential is expanded in terms of eigenfucntions in the vertical direction. Since it is a $2 \mathrm{D}$ problem, the Laplace equation can be replaced by a series of second order linear ordinary differential equations, each of which can be solved analytically. Because of the analytical expression, the higher derivatives can be obtained directly through differentiating the known functions. The method was verified with analytical solution and found to be very accurate and efficient, and was then used for a 2D floating body of arbitrary shape. Here the hybrid method is extended to the 3D problem. A major difference is that in the horizontal direction the governing 3D Laplace equation does not reduce to ordinary differential equations. 
Instead it becomes a series of 2D Helmholtz equations, each of which has to be solved through integral equations (e.g. [14]).

It is also important to ensure the continuity of velocity and pressure at the interface of the polynya and the region below the ice sheet. As expansion is already used in the vertical direction, an inner product of orthogonality can be used to enforce the required continuity [15]. Thus the computational procedure for the problem has three components: (1) a 3D integral equation in the polynya, (2) an infinite number of 2D integral equations in the region below the ice sheet, and (3) inner product on the interface to ensure continuity. This forms the major novelty of the present paper.

Apart from the development in terms of the computational technique, the present work also attempts to use the developed method to investigate the mechanism of the wave/ice sheet/body interaction. There has been extensive work on wave/ice interaction in the context of geophysics. Much of them are focused on the 2D simplified problems, for example wave propagating from open sea to the semi-infinite shore fast ice (e.g. [16-19]), across a polynya or an ice floe (e.g. [20-22]), in an infinite ice sheet but with step changes of thickness (e.g. [23]), and through one or multiple cracks (e.g. [24-26]). The methods used are generally the matched eigenfunction expansion, boundary integral equation, Wiener-Hopf technique, or the Residue Calculus approach. A recent work conducted by Mattsson, Dunham and Werpers [27] also solved the similar 2D problem through a high order finite difference method, but with the focus mainly on the acoustic waves. For an ice sheet with a complicated edge shape, the 3D effects must be considered directly through the 3D model, for example wave scatterings by a circular ice floe (e.g. [28, 29]) or an ice floe with arbitrary geometry (e.g. [30]), and by an arbitrary smooth ice polynya (e.g. [31]). As mentioned above, there has also been extensive work on wave/body interaction, such as the linear and higher order wave loads on ships and offshore structures in open sea (e.g. [32-34]), in a water channel (e.g. $[6,35])$, or in a harbor (e.g. $[36,37])$. In contrast, there have been much fewer works on the wave/ice sheet/body interaction. Typical work includes those 2D problems of a body submerged below an ice floe or floating in a polynya (e.g. [13, 38-40]), and below an ice sheet with one or multiple cracks (e.g. [41-43]). However, for 3D problems, the work has been mainly focusing on structures with simple geometries, for example bottom-mounted vertical circular cylinders in an infinitely extended ice sheet (e.g. [44, 45]) or in a circular polynya (e.g. [46]). Thus the main goal of the present work is to develop an accurate numerical solution approach for a 3D structure with a complicated shape, and undertake in depth study for the behaviour of a practical structure in the polynya surrounded by ice sheet.

The paper is organized as follows. The governing equation of the problem and corresponding 
boundary conditions are described in section 2. Formulations for the velocity potential in interior sub-domain or polynya are established in section 3.1, while those in exterior sub-domain or infinite ice covered region are given in section 3.2. In section 3.3., the solution from matching procedure on the control surface are shown. Equations for hydrodynamic loads are given in section 3.4, and numerical discretization scheme is provided in section 3.5. In section 4, verifications of the proposed method are first carried out, and then the code WISPICE is applied to a FPSO in a polynya with different shapes and floating positions. Finally, conclusions are drawn in section 5 .

\section{Mathematical Model}

The problem of flexural-gravity wave interaction with a body floating on a polynya of arbitrary shape is sketched in Fig. 1. The polynya is confined by an infinitely extended ice sheet, and the water depth of finite value $H$ is assumed to be constant. To describe the problem, a Cartesian coordinate system $O-x y z$ is defined, with $O-x y$ plane being the undisturbed mean free water surface, and $z$ axis pointing vertically upwards. The oscillation of the body is excited by a flexural-gravity incident wave, which propagates underneath the ice sheet from infinity from an angle $\beta$ with the positive $x$ axis. When the floating body is at its equilibrium position, the $z$ axis passes through the centre of mass of the body, as shown in the figure. The edge of polynya can be described parametrically by

$$
\Gamma=(x(s), y(s)) \quad(-\gamma<s<+\gamma),
$$

where $2 \gamma$ is the total arc length of the edge, and $s$ is the curvilinear coordinate along the edge.

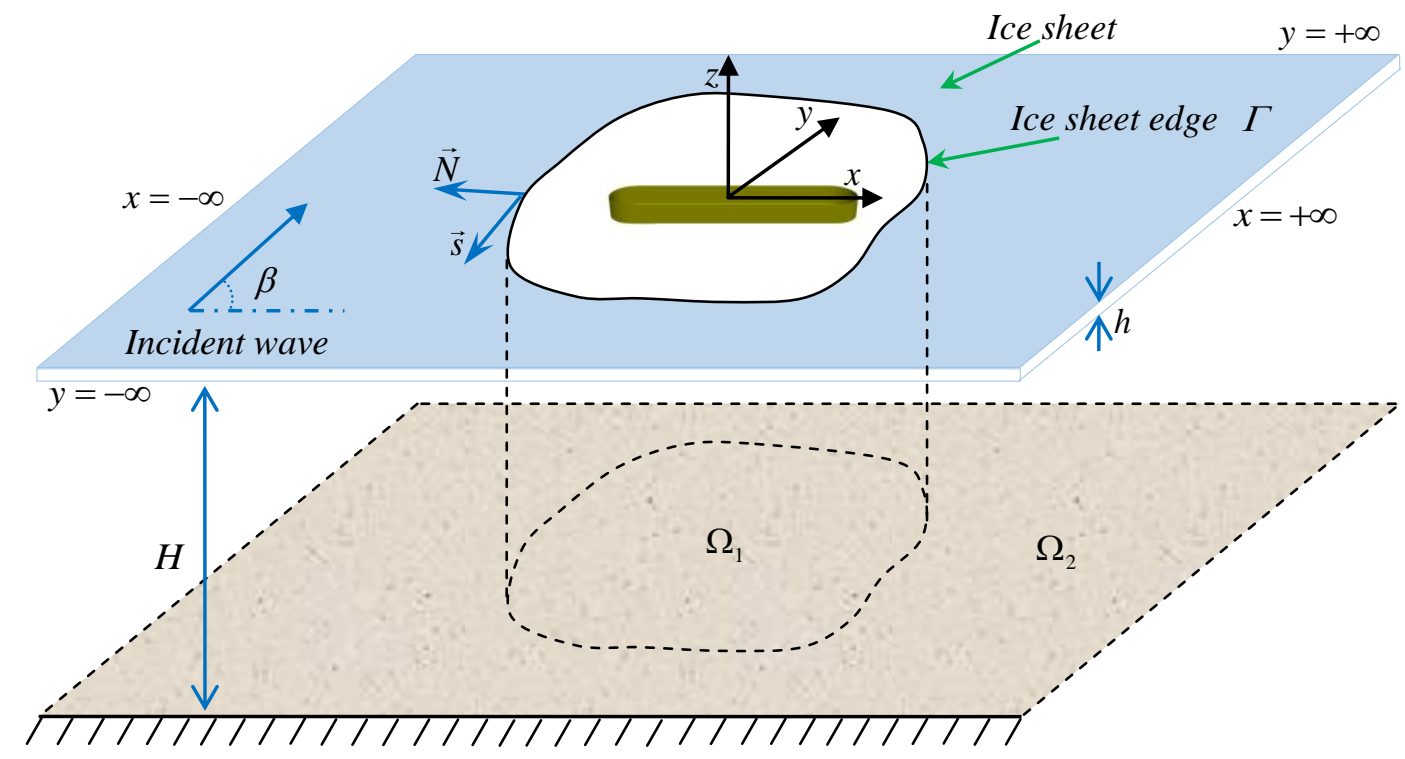

Figure 1. Coordinate system and sketch of the problem.

Based on the assumption that the fluid is inviscid, incompressible and homogeneous, and its 
motion irrotational, the velocity potential $\Phi$ can be introduced to describe the fluid flow. When the amplitude of wave motion is small compared to its length and the dimension of body, the linearization of boundary conditions can be further adopted. For sinusoidal motion in time with radian frequency $\omega$, we may write the total velocity potential as

$$
\Phi(x, y, z, t)=\operatorname{Re}\left[\eta_{0} \phi_{0}(x, y, z) \mathrm{e}^{\mathrm{i} \omega t}+\sum_{i=1}^{6} \mathrm{i} \omega \eta_{i} \phi_{i}(x, y, z) \mathrm{e}^{\mathrm{i} \omega t}\right],
$$

where $\phi_{0}=\phi_{I}+\phi_{D}$ is the scattering potential with $\phi_{I}$ as the incident potential and $\phi_{D}$ as the diffracted potential, $\eta_{0}$ is the amplitude of the incident wave; $\phi_{i}$ is the radiation potential due to the $i$-th mode of body oscillation in six degrees of freedom with complex amplitude $\eta_{i}$, i.e. $\eta_{i}$ $(i=1,2,3)$ are the translational modes along $x, y$ and $z$ directions respectively, while $\eta_{i}$ $(i=4,5,6)$ are the corresponding rotational modes. The conservation of mass requires that the velocity potential $\phi_{i}(i=0, \ldots, 6)$ should satisfy the following Laplace equation

$$
\nabla^{2} \phi_{i}+\frac{\partial^{2} \phi_{i}}{\partial z^{2}}=0
$$

throughout the fluid, where

$$
\nabla^{2}=\frac{\partial^{2}}{\partial x^{2}}+\frac{\partial^{2}}{\partial y^{2}},
$$

is the Laplacian in horizontal plane. In polynya, the combination of linearized dynamic and kinematic free surface boundary conditions provides

$$
-\omega^{2} \phi_{i}+g \frac{\partial \phi_{i}}{\partial z}=0 \quad\left(\text { in } \Omega_{1} \text { with } z=0\right),
$$

where $g$ is the acceleration due to gravity. Following Squire [10] and others, the ice sheet can be modelled as a thin elastic plate with its properties, Young's modulus $E$, Poisson's ratio $v$, density $\rho_{i}$ and thickness $h$, being assumed to be constant and its draught effect being ignored. By assuming that there is no gap between ice sheet and water upper surface, the boundary condition on their interface can be written as

$$
\left(L \nabla^{4}+\rho_{w} g-m \omega^{2}\right) \frac{\partial \phi_{i}}{\partial z}-\rho_{w} \omega^{2} \phi_{i}=0 \quad\left(\text { in } \Omega_{2} \text { with } z=0\right),
$$

where $L=E h^{3} /\left[12\left(1-v^{2}\right)\right]$ and $m=\rho_{i} h$ are respectively the effective flexural rigidity and mass per unit area of the ice sheet, $\rho_{w}$ is the density of water. On the boundary of polynya, the edge of ice sheet is assumed to be free to move, i.e. zero bending moment and shear force conditions should be satisfied, or [47]

$$
\mathcal{B}\left(\frac{\partial \phi_{i}}{\partial z}\right)=0 \quad \text { and } \mathcal{S}\left(\frac{\partial \phi_{i}}{\partial z}\right)=0 \quad((x, y) \in \Gamma, z=0),
$$

for $i=0, \ldots, 6$, where the operator $\mathcal{B}$ and $\mathcal{S}$ are respectively defined as 


$$
\begin{gathered}
\mathcal{B}=\nabla^{2}-v_{0}\left(\sin ^{2} \Theta \frac{\partial^{2}}{\partial x^{2}}+\cos ^{2} \Theta \frac{\partial^{2}}{\partial y^{2}}-\sin 2 \Theta \frac{\partial^{2}}{\partial x \partial y}\right), \\
\mathcal{S}=\frac{\partial}{\partial N} \nabla^{2}+v_{0} \frac{\partial}{\partial s}\left[\cos 2 \Theta \frac{\partial^{2}}{\partial x \partial y}+\frac{\sin 2 \Theta}{2}\left(\frac{\partial^{2}}{\partial y^{2}}-\frac{\partial^{2}}{\partial x^{2}}\right)\right],
\end{gathered}
$$

with $v_{0}=1-v$, and $\vec{N}$ and $\vec{s}$ are the unit vectors along the normal and tangential directions respectively, as shown in Fig. 1. Here, $\Theta(s)$ is the angle between positive $\vec{N}$ and $x$ axis, and thus $\vec{N}=(\cos \Theta, \sin \Theta)$ and $\vec{s}=(-\sin \Theta, \cos \Theta)$. It should be noted that in (6) and (7), the following kinematic boundary condition has been used

$$
\frac{\partial W}{\partial t}=\frac{\partial \Phi}{\partial z} \quad\left(\text { in } \Omega_{2} \text { with } z=0\right),
$$

where $W$ is the deflection of the ice sheet and can be written as

$$
W=\operatorname{Re}\left(\eta_{0} w_{0} \mathrm{e}^{\mathrm{i} \omega t}+\sum_{i=1}^{6} \mathrm{i} \omega \eta_{i} w_{i} \mathrm{e}^{\mathrm{i} \omega t}\right)
$$

Here, $w_{0}$ is the component due to the scattering potential, and $w_{i}$ is that due to the radiation potential in $i$-th mode. The impermeable condition on the mean wetted body surface $S_{B}$ can be written as

$$
\frac{\partial \phi_{i}}{\partial n}=n_{i} \text { and } \frac{\partial \phi_{D}}{\partial n}=-\frac{\partial \phi_{I}}{\partial n} \quad(i=1, \ldots, 6),
$$

where $\left(n_{1}, n_{2}, n_{3}\right)=\vec{n}$ are the components related to the translational modes, with $\vec{n}$ as the unit normal vector pointing into body, $\left(n_{4}, n_{5}, n_{6}\right)=\left(\vec{r}-\vec{r}_{0}\right) \times \vec{n}$ are those related to the rotational modes, with $\vec{r}_{0}$ as the position of the rotational centre. On the flat seabed, we have

$$
\frac{\partial \phi_{i}}{\partial z}=0 \quad(z=-H)
$$

for $i=0, \ldots, 6$. At infinity the radiation condition should be imposed, which requires that the radiated and diffracted waves should propagate outwards, or

$$
\lim _{r_{h} \rightarrow \infty} \sqrt{r_{h}}\left(\frac{\partial \phi_{i}}{\partial r_{h}}+\mathrm{i} \kappa_{0} \phi_{i}\right)=0 \text { and } \lim _{r_{h} \rightarrow \infty} \sqrt{r_{h}}\left(\frac{\partial \phi_{D}}{\partial r_{h}}+\mathrm{i} \kappa_{0} \phi_{D}\right)=0
$$

for $i=1, \ldots, 6$, where $r_{h}^{2}=x^{2}+y^{2}, \kappa_{0}$ is the flexural-gravity wave number and $\mathrm{i}=\sqrt{-1}$. It may be noticed that $\kappa_{0}$ is the purely positive real root of the dispersion equation $K_{2}\left(\omega, \kappa_{0}\right)=0$ for flexural-gravity wave in the ice sheet, with

$$
K_{2}(\omega, k) \equiv\left(L k^{4}+\rho_{w} g-m \omega^{2}\right) k \tanh (k H)-\rho_{w} \omega^{2} .
$$

Here, we may notice that the adopted linear velocity potential theory for fluid flow and the thin elastic plate model for ice sheet flexural motion have been verified extensively, e.g. as reviewed by Squire [10].

\section{Solution Procedures}


From the above section, it can be seen that the boundary condition for the velocity potential $\phi_{i}$ on the upper surface is non uniform, i.e. in polynya the free surface boundary condition (5) should be enforced, while out of polynya the ice sheet boundary condition (6) should be satisfied. To solve such a problem, we shall use the hybrid method, i.e. divide the total fluid domain into two sub-domains $\Omega_{1}$ and $\Omega_{2}$. In the former, the free surface Green function $G^{(1)}(p, q)$ will be used to construct the boundary integral equation, while in the latter, expansion in the vertical direction will be adopted. On the interface between $\Omega_{1}$ and $\Omega_{2}$, the continuity of pressure and normal velocity conditions will be enforced, and the free bending moment and shear force conditions will be imposed at the ice sheet edge.

\subsection{Boundary integral equation in polynya sub-domain $\Omega_{1}$}

In the polynya region with a free surface, we may use the following Green function [48]

$$
G^{(1)}(p, q)=\frac{1}{r_{1}}+\frac{1}{r_{2}}+2 \int_{0}^{+\infty} \mathrm{e}^{-k H} \frac{g k+\omega^{2}}{K_{1}(\omega, k)} \frac{\cosh [k(\zeta+H)]}{\cosh (k H)} \cosh [k(z+H)] J_{0}(k R) \mathrm{d} k,
$$

where the integral route from 0 to $+\infty$ should pass over the pole at $k=k_{0}$ with $K_{1}\left(\omega, k_{0}\right)=0$. Here,

$$
K_{1}(\omega, k) \equiv g k \tanh (k H)-\omega^{2},
$$

is the dispersion equation for free surface gravity wave, $r_{1}$ is the distance between $p$ and $q$, $r_{2}$ is the distance between $p$ and the mirror image of $q$ about the flat seabed, $J_{0}(k R)$ is the zero order Bessel function of first kind [49], with $R$ as the horizontal distance between $p$ and $q$. We may note that (16) can be also written into the series form as [48]

$$
G^{(1)}(p, q)=-4 \pi \mathrm{i} \sum_{m=0}^{\infty} D_{m} \cosh ^{2}\left(k_{m} H\right) Z_{m}(z) Z_{m}(\zeta) H_{0}^{(2)}\left(k_{m} R\right),
$$

where $H_{0}^{(2)}\left(k_{m} R\right)$ is the Hankel function of second kind [49],

$$
\begin{gathered}
D_{m}=\frac{k_{m}}{2 k_{m} H+\sinh \left(2 k_{m} H\right)}, \\
Z_{m}(z)=\frac{\cosh \left[k_{m}(z+H)\right]}{\cosh \left(k_{m} H\right)},
\end{gathered}
$$

with $k_{m}$ as the root of the dispersion equation in (17). It should be noticed that $k_{0}$ is the purely positive real root, and $k_{m}(m=1, \ldots, \infty)$ are an infinite number of purely negative imaginary roots. It may be noticed that the series of $Z_{m}(z)$ is orthogonal [2] and a velocity potential can be expanded into a series of these functions in the vertical direction.

Applying Green's identity to $G^{(1)}$ and $\phi_{i}$, we have

$$
\alpha^{(1)}(p) \phi_{i}(p)=\int_{S}\left[G^{(1)}(p, q) \frac{\partial \phi_{i}(q)}{\partial n}-\frac{\partial G^{(1)}(p, q)}{\partial n} \phi_{i}(q)\right] \mathrm{d} s \quad(i=0, \ldots, 6),
$$


1 where both the normal derivative and integration are carried out with respect to the source point $2 q, \alpha^{(1)}(p)$ is the solid angle at point $p$, and $S$ is comprised of all the boundaries of the 3 sub-domain $\Omega_{1}$. It should be noticed that inclusion or removal of the imaginary part of $G^{(1)}$ in 4 (16) would not affect (21). As the imaginary part is nonsingular, its integration with $\phi_{i}$ through 5 Green's identity over the closed boundary $S_{1}$ of $\Omega_{1}$ is zero. Invoking the boundary conditions satisfied by $G^{(1)}$ and $\phi_{i}$, we have that only the integrals over the mean wetted body surface $S_{B}$ and the interface $S_{C}$ are nonzero. Then the integral equation (21) can be further written as

$$
\alpha^{(1)}(p) \phi_{i}(p)=\int_{S_{B}+S_{C}}\left[G^{(1)}(p, q) \frac{\partial \phi_{i}(q)}{\partial n}-\frac{\partial G^{(1)}(p, q)}{\partial n} \phi_{i}(q)\right] \mathrm{d} s .
$$

It might be noticed that when $S_{C}$ is removed from (22) there will be irregular frequencies for the Neumann problem exterior the body, which correspond to the eigensolutions of the interior Dirichlet problem. However, the irregular frequencies without $S_{C}$ will not be irregular frequencies here. On the interface $S_{C}$, which is vertically extended from the ice edge to the seabed, we may expand $\phi_{i}$ and its normal derivative $\partial \phi_{i} / \partial n$ into a series of orthogonal functions $Z_{m}(z)$ in $(20)$ or

$$
\phi_{i}(p)=\sum_{m=0}^{\infty} \phi_{i, m}(x, y) Z_{m}(z), \frac{\partial \phi_{i}(p)}{\partial n}=\sum_{m=0}^{\infty} \frac{\partial \phi_{i, m}(x, y)}{\partial n} Z_{m}(z) .
$$

Here, it may be noticed that since $S_{C}$ is a vertical surface, the normal vector $\vec{n}$ is independent of $z$, or it is the same as that of $\Gamma$ defined below (9). Substituting (23) into (22), we have

$$
\begin{aligned}
\alpha^{(1)}(p) \phi_{i}(p)= & \int_{S_{B}}\left[G^{(1)}(p, q) \frac{\partial \phi_{i}(q)}{\partial n}-\frac{\partial G^{(1)}(p, q)}{\partial n} \phi_{i}(q)\right] \mathrm{d} s \\
& +\sum_{m=0}^{\infty} \int_{\Gamma}\left[U_{m}(p, \xi, \eta) \frac{\partial \phi_{i, m}(\xi, \eta)}{\partial N}-V_{m}(p, \xi, \eta) \phi_{i, m}(\xi, \eta)\right] \mathrm{d} l
\end{aligned},
$$

where

$$
\begin{gathered}
U_{m}(p, \xi, \eta)=\int_{-H}^{0}\left[G^{(1)}(p, q) Z_{m}(\zeta)\right] \mathrm{d} \zeta, \\
V_{m}(p, \xi, \eta)=\int_{-H}^{0}\left[\frac{\partial G^{(1)}(p, q)}{\partial N} Z_{m}(\zeta)\right] \mathrm{d} \zeta .
\end{gathered}
$$

Substituting the series form of $G^{(1)}$ in (18) into the above two equations, and noticing the orthogonality of the vertical modes $Z_{m}(z)$, we have

$$
\begin{gathered}
U_{m}(p, \xi, \eta)=-\pi \mathrm{i} Z_{m}(z) H_{0}^{(2)}\left(k_{m} R\right), \\
V_{m}(p, \xi, \eta)=-\pi \mathrm{i} Z_{m}(z) \frac{\partial H_{0}^{(2)}\left(k_{m} R\right)}{\partial N} .
\end{gathered}
$$

When the field point $p$ is located on the interface $S_{C}$, we may also replace $\phi_{i}(p)$ on the left hand side of (24) with (23). Then multiplying both sides of the obtained results with $Z_{\tilde{m}}(z)$, and integrating with respect to $z$ from $-H$ to 0 , we have 


$$
\begin{aligned}
\frac{C_{\tilde{m}}}{\pi} \mathrm{i} \alpha^{(1)} \phi_{i, \tilde{m}}(x, y)= & \int_{S_{B}}\left[Z_{\tilde{m}}(\zeta) H_{0}^{(2)}\left(k_{\tilde{m}} R\right) \frac{\partial \phi_{i}(q)}{\partial n}-\frac{\partial Z_{\tilde{m}}(\zeta) H_{0}^{(2)}\left(k_{\tilde{m}} R\right)}{\partial n} \phi_{i}(q)\right] \mathrm{d} s \\
& +C_{\tilde{m}} \int_{\Gamma}\left[H_{0}^{(2)}\left(k_{\tilde{m}} R\right) \frac{\partial \phi_{i, \tilde{m}}(\xi, \eta)}{\partial N}-\frac{\partial H_{0}^{(2)}\left(k_{\tilde{m}} R\right)}{\partial N} \phi_{i, \tilde{m}}(\xi, \eta)\right] \mathrm{d} l
\end{aligned}
$$

2

where

$$
C_{\tilde{m}}=\int_{-H}^{0} Z_{\tilde{m}}(z) Z_{\tilde{m}}(z) \mathrm{d} z=\left[\frac{H}{2}+\frac{\sinh \left(2 k_{\tilde{m}} H\right)}{4 k_{\tilde{m}}}\right] / \cosh ^{2}\left(k_{\tilde{m}} H\right) .
$$

\subsection{Series expansion in the ice covered sub-domain $\Omega_{2}$}

In the ice covered sub-domain $\Omega_{2}$, through using variable separation method, we have

$$
\phi_{i}(p)=\sum_{m} \varphi_{i, m}(x, y) \psi_{m}(z)
$$

where the summation contains all possible eigen values and nonzero eigenfunctions, with

$$
\nabla^{2} \varphi_{i, m}+\kappa_{m}^{2} \varphi_{i, m}=0
$$

and

$$
\frac{\mathrm{d}^{2} \psi_{m}}{\mathrm{~d} z^{2}}-\kappa_{m}^{2} \psi_{m}=0
$$

From (33), (6) and (13), we have

$$
\psi_{m}(z)=\frac{\cosh \left[\kappa_{m}(z+H)\right]}{\cosh \left(\kappa_{m} H\right)},
$$

where $\kappa_{m}$ are the roots of the dispersion equation in (15). It may be noticed that $\kappa_{-2}$ and $\kappa_{-1}$ are two complex roots with negative imaginary parts and symmetric about the imaginary axis, $\kappa_{0}$ is the purely positive real root, $\kappa_{m}(m=1, \ldots, \infty)$ is an infinite number of purely negative imaginary roots. Thus (31) becomes

$$
\phi_{i}(p)=\delta_{0, i} \phi_{I}(p)+\sum_{m=-2}^{\infty} \varphi_{i, m}(x, y) \psi_{m}(z)
$$

where $\delta_{0, i}=1$ if $i=0$ and $\delta_{0, i}=0$ for others. The incident velocity potential in (35) can be given as

$$
\phi_{I}(p)=\varphi_{I}(x, y) \psi_{0}(z)
$$

where

$$
\varphi_{I}(x, y)=\mathrm{Ie}^{-\mathrm{i} \kappa_{0}(x \cos \beta+y \sin \beta)},
$$

with $I=\mathrm{i} g / \omega$.

Here, it may be noted that Bennetts and Williams [31] used the method of vertical modes, or the velocity potential is expanded into a series of vertical eigenfunctions which are the same as those in (34). Then the functions of $\kappa_{-2}$ and $\kappa_{-1}$ can be written in terms of others, i.e. the summation in (31) starts from $m=0$ and the governing equations for $\varphi_{i, m}$ are coupled. Here, we follow the 
normal variable separation procedure and take into account all the eigen values. The governing equation for each $\varphi_{i, m}$ in (32) is independent. To solve the Helmholtz equation in (32), together with the radiation condition in (14), we may use the Green function [14]

$$
G^{(2)}(p, q)=\frac{\pi}{2 \mathrm{i}} H_{0}^{(2)}\left(\kappa_{m} R\right)
$$

Applying Green's identity to $G^{(2)}(p, q)$ and $\varphi_{i, m}$, we have along the ice sheet edge

$$
\alpha^{(2)}(p) \varphi_{i, m}(p)=\int_{\Gamma}\left[G^{(2)}(p, q) \frac{\partial \varphi_{i, m}(q)}{\partial N}-\frac{\partial G^{(2)}(p, q)}{\partial N} \varphi_{i, m}(q)\right] \mathrm{d} l \quad(i=0, \ldots, 6),
$$

where $\alpha^{(2)}$ is the two dimensional solid angle at point $p$. Here, for $i=0$, the incident velocity potential should be excluded. It may be noticed that since both $G^{(2)}$ and $\varphi_{i, m}$ satisfy the radiation condition in (14), the integral along the circle of infinite radius has been removed.

It should be noticed when expressing $\varphi_{i, m}$ by $\partial \varphi_{i, m} / \partial N$ through solving the boundary integral equation (39), irregular frequencies may appear. To avoid this or obtain a unique relationship between $\varphi_{i, m}$ and $\partial \varphi_{i, m} / \partial N$, (39) is added by a hypersingular integral equation [50]

$$
\beta \alpha^{(2)}(p) \frac{\partial \varphi_{i, m}(p)}{\partial N_{p}}=\beta \int_{\Gamma}\left[\frac{\partial G^{(2)}(p, q)}{\partial N_{p}} \frac{\partial \varphi_{i, m}(q)}{\partial N}-\frac{\partial^{2} G^{(2)}(p, q)}{\partial N_{p} \partial N} \varphi_{i, m}(q)\right] \mathrm{d} l .
$$

Here, $\beta$ is a coupling constant that can be chosen as $\mathrm{i} /\left|\kappa_{m}\right|[51]$.

\subsection{Matching on the interface between the two sub-domains}

On the interface $S_{C}$ between the two sub-domains, both the pressure and normal velocity should be continuous, i.e.
where the superscripts (1) and (2) indicate that the velocity potential and its normal derivative are for the sub-domain $\Omega_{1}$ and $\Omega_{2}$ respectively. To satisfy the conditions in (41) and (42), we may use the following inner product [15]

$$
<\psi_{m}, \psi_{\tilde{m}}>=\int_{-H}^{0} \psi_{m} \psi_{\tilde{m}} \mathrm{~d} z+\frac{L}{\rho_{w} \omega^{2}}\left(\frac{\partial \psi_{m}}{\partial z} \frac{\partial^{3} \psi_{\tilde{m}}}{\partial z^{3}}+\frac{\partial^{3} \psi_{m}}{\partial z^{3}} \frac{\partial \psi_{\tilde{m}}}{\partial z}\right)_{z=0}
$$

Then $<\psi_{m}, \psi_{\tilde{m}}>=0$ if $m \neq \tilde{m}$, and $<\psi_{m}, \psi_{\tilde{m}}>=Q_{m}$ if $m=\tilde{m}$, with

$$
Q_{m}=\frac{2 \kappa_{m} H+\sinh \left(2 \kappa_{m} H\right)}{4 \kappa_{m} \cosh ^{2}\left(\kappa_{m} H\right)}+\frac{2 L \kappa_{m}^{4}}{\rho_{w} \omega^{2}} \tanh ^{2}\left(\kappa_{m} H\right) .
$$

To satisfy the continuity condition of pressure in (41), applying the inner product to $\phi_{i}^{(2)}$ and $\psi_{\tilde{m}}$, we have 


$$
\begin{aligned}
<\phi_{i}^{(2)}, \psi_{\tilde{m}}> & =\int_{-H}^{0} \phi_{i}^{(2)} \psi_{\tilde{m}} \mathrm{~d} z+\frac{L}{\rho_{w} \omega^{2}}\left(\frac{\partial \phi_{i}^{(2)}}{\partial z} \frac{\partial^{3} \psi_{\tilde{m}}}{\partial z^{3}}+\frac{\partial^{3} \phi_{i}^{(2)}}{\partial z^{3}} \frac{\partial \psi_{\tilde{m}}}{\partial z}\right)_{z=0} \\
& =\int_{-H}^{0} \phi_{i}^{(1)} \psi_{\tilde{m}} \mathrm{~d} z+\frac{L}{\rho_{w} \omega^{2}}\left(\frac{\partial \phi_{i}^{(2)}}{\partial z} \frac{\partial^{3} \psi_{\tilde{m}}}{\partial z^{3}}+\frac{\partial^{3} \phi_{i}^{(2)}}{\partial z^{3}} \frac{\partial \psi_{\tilde{m}}}{\partial z}\right)_{z=0}
\end{aligned}
$$

2 Similarly, we have for the continuity condition of the normal velocity

3

$$
\begin{aligned}
<\frac{\partial \phi_{i}^{(2)}}{\partial n}, \psi_{\tilde{m}}> & =\int_{-H}^{0} \frac{\partial \phi_{i}^{(2)}}{\partial n} \psi_{\tilde{m}} \mathrm{~d} z+\frac{L}{\rho_{w} \omega^{2}}\left(\frac{\partial^{2} \phi_{i}^{(2)}}{\partial z \partial N} \frac{\partial^{3} \psi_{\tilde{m}}}{\partial z^{3}}+\frac{\partial^{4} \phi_{i}^{(2)}}{\partial z^{3} \partial N} \frac{\partial \psi_{\tilde{m}}}{\partial z}\right)_{z=0} \\
& =\int_{-H}^{0} \frac{\partial \phi_{i}^{(1)}}{\partial n} \psi_{\tilde{m}} \mathrm{~d} z+\frac{L}{\rho_{w} \omega^{2}}\left(\frac{\partial^{2} \phi_{i}^{(2)}}{\partial z \partial N} \frac{\partial^{3} \psi_{\tilde{m}}}{\partial z^{3}}+\frac{\partial^{4} \phi_{i}^{(2)}}{\partial z^{3} \partial N} \frac{\partial \psi_{\tilde{m}}}{\partial z}\right)_{z=0}
\end{aligned}
$$

Substituting equation (35) for $\phi_{i}^{(2)}$ respectively into the left hand sides of (45) and (46), we have

$$
\left(\delta_{0, i} \delta_{0, \tilde{m}} \varphi_{I}+\varphi_{i, \tilde{m}}\right) Q_{\tilde{m}}=\int_{-H}^{0} \phi_{i}^{(1)} \psi_{\tilde{m}} \mathrm{~d} z+\frac{L}{\rho_{w} \omega^{2}}\left(\frac{\partial \phi_{i}^{(2)}}{\partial z} \frac{\partial^{3} \psi_{\tilde{m}}}{\partial z^{3}}+\frac{\partial^{3} \phi_{i}^{(2)}}{\partial z^{3}} \frac{\partial \psi_{\tilde{m}}}{\partial z}\right)_{z=0},
$$

and

$$
\left(\delta_{0, i} \delta_{0, \tilde{m}} \frac{\partial \varphi_{I}}{\partial N}+\frac{\partial \varphi_{i, \tilde{m}}}{\partial N}\right) Q_{\tilde{m}}=\int_{-H}^{0} \frac{\partial \phi_{i}^{(1)}}{\partial n} \psi_{\tilde{m}} \mathrm{~d} z+\frac{L}{\rho_{w} \omega^{2}}\left(\frac{\partial^{2} \phi_{i}^{(2)}}{\partial z \partial N} \frac{\partial^{3} \psi_{\tilde{m}}}{\partial z^{3}}+\frac{\partial^{4} \phi_{i}^{(2)}}{\partial z^{3} \partial N} \frac{\partial \psi_{\tilde{m}}}{\partial z}\right)_{z=0} .
$$

By noticing equation (3) or $\partial^{2} \phi_{i} / \partial z^{2}=-\nabla^{2} \phi_{i}$, we may rewrite the above two equations as

$$
\left(\delta_{0, i} \delta_{0, \tilde{m}} \varphi_{I}+\varphi_{i, \tilde{m}}\right) Q_{\tilde{m}}=\int_{-H}^{0} \phi_{i}^{(1)} \psi_{\tilde{m}} \mathrm{~d} z+\frac{L}{\rho_{w} \omega^{2}}\left[\frac{\partial \phi_{i}^{(2)}}{\partial z} \frac{\partial^{3} \psi_{\tilde{m}}}{\partial z^{3}}-\nabla^{2}\left(\frac{\partial \phi_{i}^{(2)}}{\partial z}\right) \frac{\partial \psi_{\tilde{m}}}{\partial z}\right]_{z=0},
$$

and

$$
\left(\delta_{0, i} \delta_{0, \tilde{m}} \frac{\partial \varphi_{I}}{\partial N}+\frac{\partial \varphi_{i, \tilde{m}}}{\partial N}\right) Q_{\tilde{m}}=\int_{-H}^{0} \frac{\partial \phi_{i}^{(1)}}{\partial n} \psi_{\tilde{m}} \mathrm{~d} z+\frac{L}{\rho_{w} \omega^{2}}\left\{\frac{\partial^{2} \phi_{i}^{(2)}}{\partial z \partial N} \frac{\partial^{3} \psi_{\tilde{m}}}{\partial z^{3}}-\left[\frac{\partial}{\partial N} \nabla^{2}\left(\frac{\partial \phi_{i}^{(2)}}{\partial z}\right)\right] \frac{\partial \psi_{\tilde{m}}}{\partial z}\right\}_{z=0}
$$

Using

$$
\frac{\partial x}{\partial s}=-\sin \Theta \text { and } \frac{\partial y}{\partial s}=\cos \Theta
$$

we can write the operators $\mathcal{B}$ and $\mathcal{S}$ in (8) and (9) respectively as

$$
\begin{gathered}
\mathcal{B}=\nabla^{2}-v_{0}\left(\frac{\partial^{2}}{\partial s^{2}}+\frac{\partial \Theta}{\partial s} \frac{\partial}{\partial N}\right), \\
\mathcal{S}=\frac{\partial}{\partial N} \nabla^{2}+v_{0} \frac{\partial}{\partial s}\left(\frac{\partial^{2}}{\partial s \partial N}-\frac{\partial \Theta}{\partial s} \frac{\partial}{\partial s}\right) .
\end{gathered}
$$

It should be noticed that in (52) and (53), the partial derivatives with respect to $s$ are carried out with respect to the curvilinear coordinate, instead of the tangential direction. For the first order derivative, the former is the same as the latter. However, this may not be the case for higher order derivatives. Invoking the ice sheet edge condition in (7), we then have

$$
\begin{gathered}
\nabla^{2}\left(\frac{\partial \phi_{i}^{(2)}}{\partial z}\right)=v_{0}\left(\frac{\partial^{2}}{\partial s^{2}}+\frac{\partial \Theta}{\partial s} \frac{\partial}{\partial N}\right)\left(\frac{\partial \phi_{i}^{(2)}}{\partial z}\right) \\
\frac{\partial}{\partial N} \nabla^{2}\left(\frac{\partial \phi_{i}^{(2)}}{\partial z}\right)=-v_{0} \frac{\partial}{\partial s}\left(\frac{\partial^{2}}{\partial s \partial N}-\frac{\partial \Theta}{\partial s} \frac{\partial}{\partial s}\right)\left(\frac{\partial \phi_{i}^{(2)}}{\partial z}\right) .
\end{gathered}
$$



and (23), we have

$$
\left(\delta_{0, i} \delta_{0, \tilde{m}} \varphi_{I}+\varphi_{i, \tilde{m}}\right) Q_{\tilde{m}}=\sum_{m=0}^{\infty} \phi_{i, m} E_{m, \tilde{m}}+\sum_{m=-2}^{\infty} f_{m, \tilde{m}}^{P} \varphi_{i, m}+\delta_{0, i} f_{0, \tilde{m}}^{P} \varphi_{I},
$$

and

$$
\left(\delta_{0, i} \delta_{0, \tilde{m}} \frac{\partial \varphi_{I}}{\partial N}+\frac{\partial \varphi_{i, \tilde{m}}}{\partial N}\right) Q_{\tilde{m}}=\sum_{m=0}^{\infty} \frac{\partial \phi_{i, m}}{\partial N} E_{m, \tilde{m}}+\sum_{m=-2}^{\infty} f_{m, \tilde{m}}^{V} \varphi_{i, m}+\delta_{0, i} f_{0, \tilde{m}}^{V} \varphi_{I}
$$

where the operators $f_{m, \tilde{m}}^{P}$ and $f_{m, \tilde{m}}^{V}$ are respectively defined as

$$
\begin{gathered}
f_{m, \tilde{m}}^{P}=\frac{L}{\rho_{w} \omega^{2}} \frac{\partial \psi_{m}}{\partial z}\left[\left(\frac{\partial^{3} \psi_{\tilde{m}}}{\partial z^{3}}-v_{0} \frac{\partial \psi_{\tilde{m}}}{\partial z} \frac{\partial^{2}}{\partial s^{2}}\right)-v_{0} \frac{\partial \psi_{\tilde{m}}}{\partial z} \frac{\partial \Theta}{\partial s} \frac{\partial}{\partial N}\right]_{z=0}, \\
f_{m, \tilde{m}}^{V}=\frac{L}{\rho_{w} \omega^{2}} \frac{\partial \psi_{m}}{\partial z}\left[\left(\frac{\partial^{3} \psi_{\tilde{m}}}{\partial z^{3}}+v_{0} \frac{\partial \psi_{\tilde{m}}}{\partial z} \frac{\partial^{2}}{\partial s^{2}}\right) \frac{\partial}{\partial N}-v_{0} \frac{\partial \psi_{\tilde{m}}}{\partial z}\left(\frac{\partial^{2} \Theta}{\partial s^{2}} \frac{\partial}{\partial s}+\frac{\partial \Theta}{\partial s} \frac{\partial^{2}}{\partial s^{2}}\right)\right]_{z=0},
\end{gathered}
$$

and

$$
E_{m, \tilde{m}}=\int_{-H}^{0} Z_{m} \psi_{\tilde{m}} \mathrm{~d} z=\frac{1}{\cosh \left(k_{m} H\right) \cosh \left(\kappa_{\tilde{m}} H\right)}\left\{\frac{\sinh \left[H\left(k_{m}-\kappa_{\tilde{m}}\right)\right]}{2\left(k_{m}-\kappa_{\tilde{m}}\right)}+\frac{\sinh \left[H\left(k_{m}+\kappa_{\tilde{m}}\right)\right]}{2\left(k_{m}+\kappa_{\tilde{m}}\right)}\right\} .
$$

It may be noticed that in equations (56) and (57), there exist up to second order partial derivatives with respect to the curvilinear coordinate $s$, which can be obtained numerically through the five point finite difference method [52], or

$$
\begin{gathered}
\frac{\partial f_{i}}{\partial s}=\frac{1}{12 \Delta s}\left(f_{i-2}-8 f_{i-1}+8 f_{i+1}-f_{i+2}\right), \\
\frac{\partial^{2} f_{i}}{\partial s^{2}}=\frac{1}{12 \Delta s^{2}}\left(-f_{i-2}+16 f_{i-1}-30 f_{i}+16 f_{i+1}-f_{i+2}\right),
\end{gathered}
$$

where $\Delta s$ is the equally spaced arc length between the two adjacent points.

\subsection{Hydrodynamic coefficients and wave exciting force}

After the velocity potentials in sub-domains $\Omega_{1}$ and $\Omega_{2}$ have been solved, we can obtain the pressure at any point in fluid through the linear Bernoulli equation. Then the hydrodynamic force exerting on the body can be computed through integrating the pressure over the body surface. Invoking the decomposition of the velocity potential in equation (2), we may divide the total force into two parts, i.e. the radiation force due to the forced body oscillation, and the wave exciting force due to the incident and diffracted potentials [2]. For the radiation force, we may write it in form of added mass $\mu_{j k}$ and damping coefficient $\lambda_{j k}$ as

$$
\mu_{j k}-\mathrm{i} \frac{\lambda_{j k}}{\omega}=\rho_{w} \int_{S_{B}} \phi_{k} n_{j} \mathrm{~d} s
$$

while for the wave exciting force $f_{E, j}$, we have

$$
f_{E, j}=-\mathrm{i} \omega \rho_{w} \int_{S_{B}} \phi_{0} n_{j} \mathrm{~d} s .
$$




\subsection{Numerical solution through discretization and truncation}

To solve the velocity potentials $\phi_{i}$ numerically, the complex body surface $S_{B}$ is divided into $N_{B}$ flat panels, while the curved ice sheet edge $\Gamma$ is divided into $N_{\Gamma}$ straight line segments. On each panel or segment, the potentials $\phi_{i}$ or $\phi_{i, m}$ and $\varphi_{i, m}$ are assumed to be constant, and the conditions are imposed at the centre of each panel. This means that the solid angle $\alpha_{1}$ in the integral equation (21) can always be taken to be $2 \pi$, and the solid angle $\alpha_{2}$ in the integral equation (39) can always be taken to be $-\pi$, where minus sign is due to the fact that the normal vector is pointing from free surface into the ice sheet. For the infinite series in (23) and (35), the upper bound is truncated at $M-1$ and $M-3$ respectively. Therefore, we will have $N_{B}+4 N_{\Gamma} \times M$ unknowns. From (24) and (29) we can obtain $N_{B}+N_{\Gamma} \times M$ equations, while from equation (39) we have another $N_{\Gamma} \times M$ equations. The continuity requirement in (56) and (57) will provide additional $2 N_{\Gamma} \times M$ equations. This makes a total of $N_{B}+4 N_{\Gamma} \times M$ equations, which is the same as the number of unknowns.

The Hankel function used in (39) contains a logarithmic singularity as the source point approaches to the field point, or

$$
H_{0}^{(2)}(k R) \rightarrow \frac{2}{\pi \mathrm{i}} \ln (R) \text { as } R \rightarrow 0 .
$$

Thus, when conducting the integration of the Hankel function over a line element, we may subtract the singular term from the integral

$$
H_{0}^{(2)}(k R)=\left[H_{0}^{(2)}(k R)-\frac{2}{\pi \mathrm{i}} \ln (R)\right]+\frac{2}{\pi \mathrm{i}} \ln (R),
$$

The integration of the first term can be carried out through the standard numerical scheme, while the second term can be evaluated analytically [2].

\section{Numerical Results}

In this section, a bottom mounted vertical circular cylinder in a circular polynya is first studied to verify the developed method. Then the code WISPICE is used for obtaining the hydrodynamic loads on a FPSO, which is a realistic offshore structure rather than an idealized geometry, floating in a polynya. In following numerical computations, the typical parameters of ice sheet are taken to be [8]

$$
E=5 \mathrm{GPa}, \quad v=0.3, \rho_{i}=922.5 \mathrm{~kg} \mathrm{~m}^{-3},
$$

to provide physical meaningful results. All the results will be presented in the dimensionless form based on the basic parameters, i.e. density of water $\rho_{w}=1025 \mathrm{~kg} \mathrm{~m}^{-3}$, acceleration due to gravity $g=9.80 \mathrm{~m} \mathrm{~s}^{-2}$, and a characteristic length scale.

\subsection{Verification: a bottom mounted vertical circular cylinder in a circular polynya}


We first consider the wave radiation and diffraction problems of a circular cylinder with radius $a$ standing in a circular polynya with radius $c$. The centre of cylinder coincides with that of polynya. For the rotational motion, it is assumed that the cylinder is articulated at the bottom or $\vec{r}_{0}=(0,0,-H)$ and allowed to rotate in the $O x z$ plane, as in Drake, Eatock Taylor and Matsui [53]. The case has been investigated by Ren, Wu and Ji [46] analytically, through the matched eigenfunction expansions. To demonstrate the accuracy and efficiency of the present hybrid method, we adopt the same parameters as those in Ren, Wu and Ji [46], and the radius of cylinder $a$ is chosen as the characteristic length scale, i.e. $a=1, c=3, H=10, h=0.1$. The incident flexural-gravity wave angle is set to be $\beta=0$. There are 1960 panels on the body surface, and 100 line segments on the ice sheet edge, with the discretization being shown in Fig. 2. The infinite series of the eigenfunction expansions is truncated at $M=50$. These have been found to be sufficient for convergence based on the convergence study similar to that in $\mathrm{Shi}, \mathrm{Li}$ and $\mathrm{Wu}$ [36]. Fig. 3 shows the added mass $\mu_{i j}$ and damping coefficient $\lambda_{i j}$ against wave number $k_{0}$, together with the analytical results from Ren, Wu and Ji [46] denoted by dashed lines. The corresponding wave exciting force in surge and pitch are given in Fig. 4. It can be seen from these two figures that further increasing $N_{B}, N_{\Gamma}$ and $M$ will give graphically indistinguishable results, and the convergent numerical results are in excellent agreement with those analytical solutions. This shows that the present hybrid method is accurate for wave radiation and diffraction problems of a structure in a polynya. It means that results at a desired accuracy can be achieved through refining the mesh with larger $N_{B}$ and $N_{\Gamma}$, and increasing the number of terms $M$ kept in the infinite series.

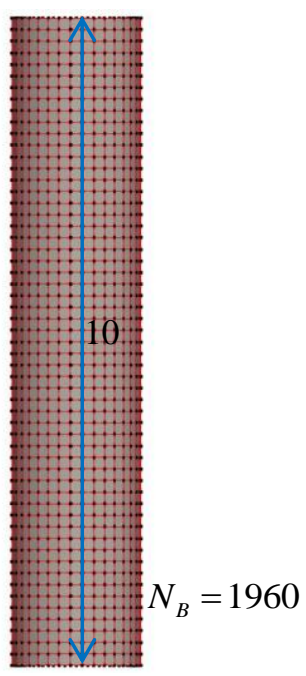

(a)

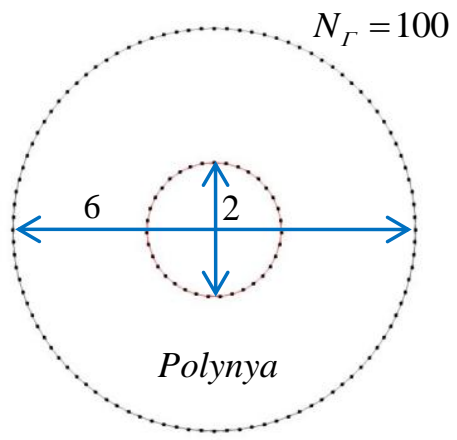

(b)

Fig. 2. The mesh on the body surface and ice sheet edge. (a) on the body; $(b)$ on the ice sheet edge. 


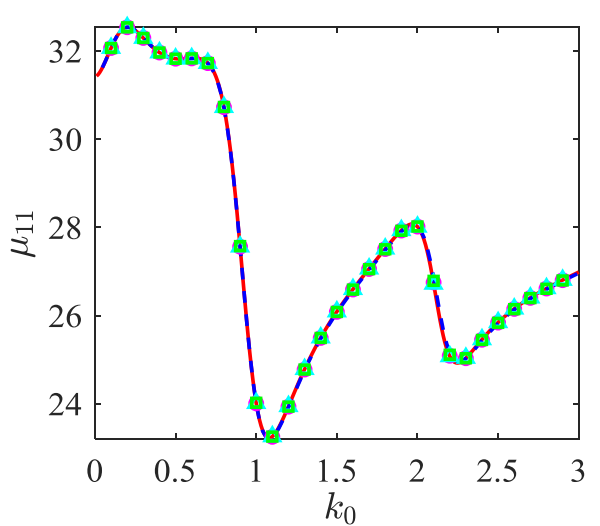

(a)

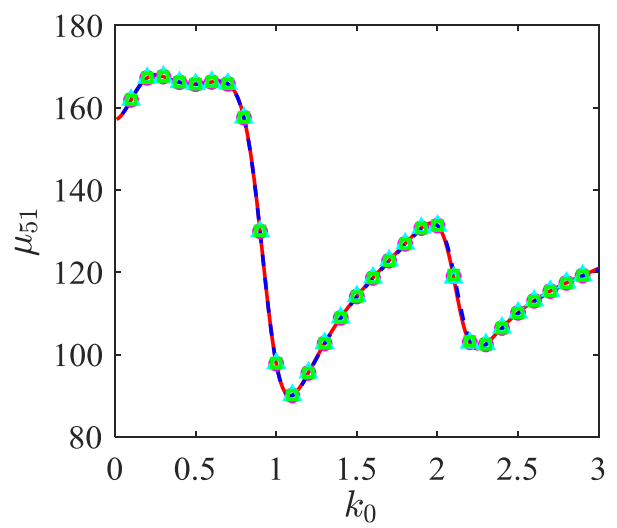

(c)

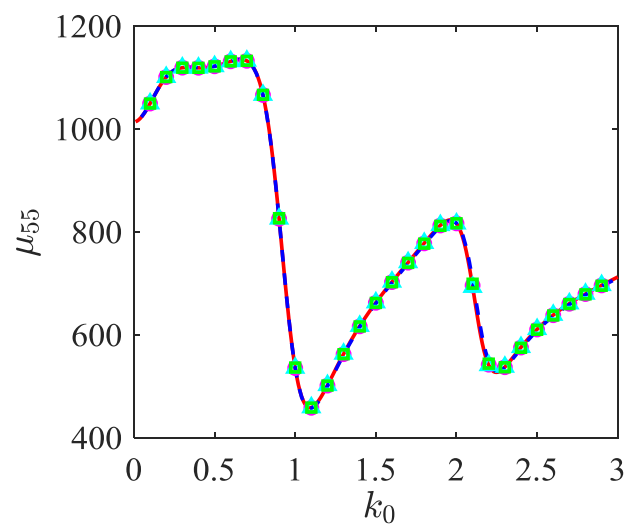

(e)

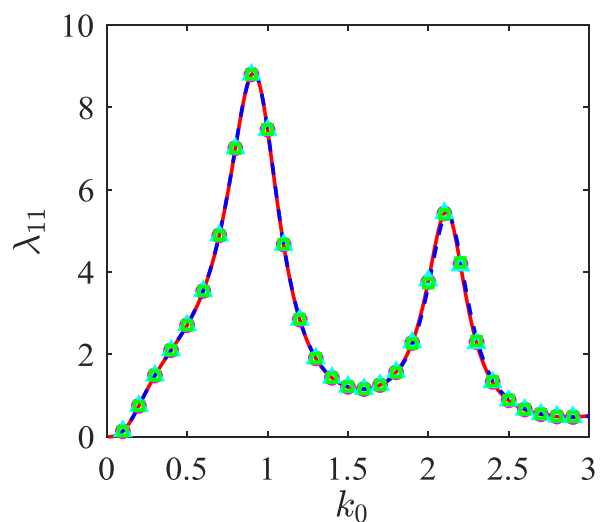

(b)

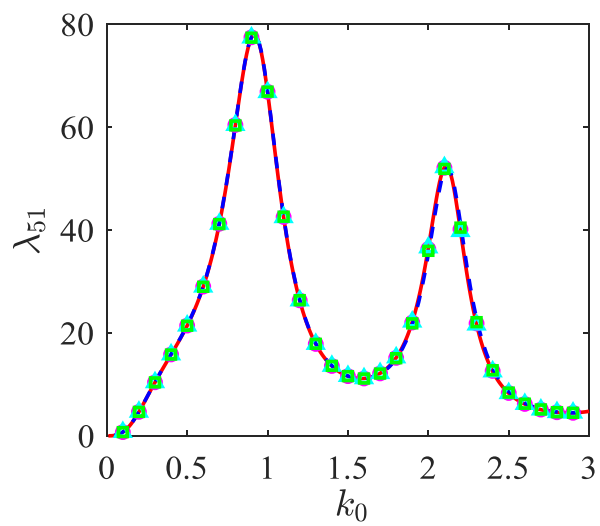

(d)

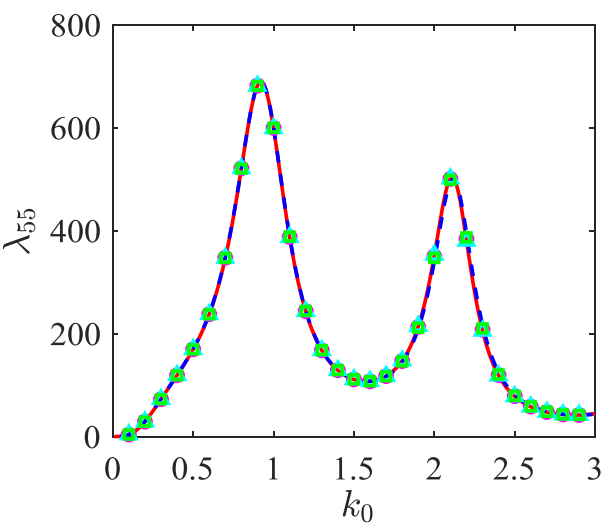

$(f)$

Fig. 3. Added mass $\mu_{j k}$ and damping coefficient $\lambda_{j k}$ of a circular cylinder in a circular polynya against wave number $k_{0}$. Solid lines: numerical results by the present hybrid method $\left(N_{B}=1960, N_{\Gamma}=100, M=50\right)$; dashed lines: analytical solutions from Fig. 4 of Ren, Wu and Ji [46]; circles: finer mesh on the body surface with $N_{B}=2924$; triangles: finer mesh on the ice sheet edge with $N_{\Gamma}=150$; squares: more terms kept in the series with $M=75 .(a=1, c=3, H=10, h=0.1, \quad m=0.09, L=4.5582, \beta=0)$ 


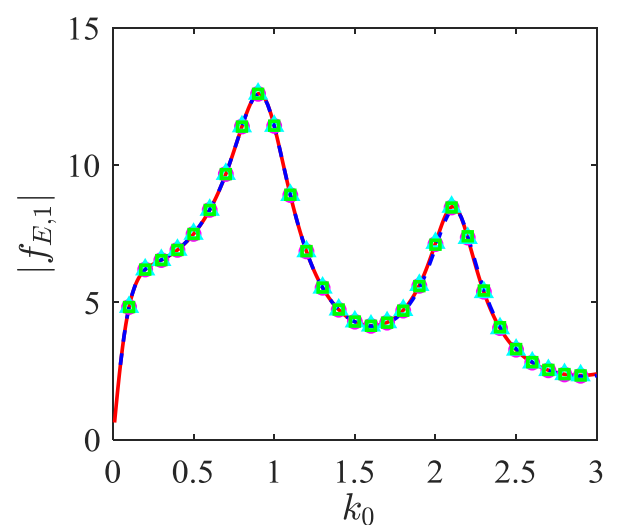

(a)

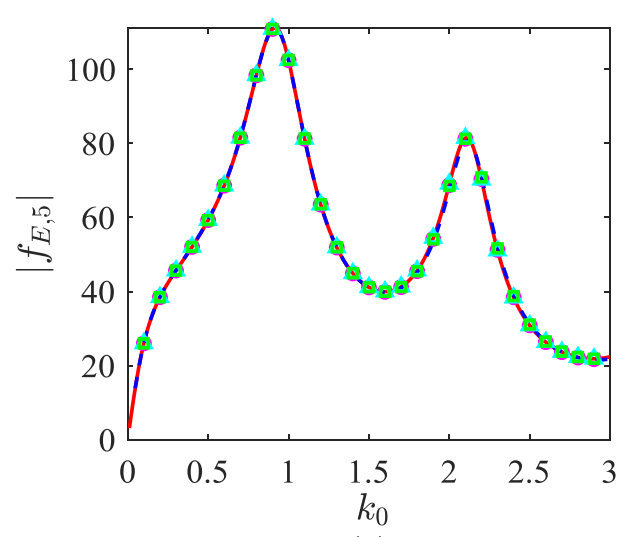

(b)
3

Fig. 4. Wave exciting force $f_{E, j}$ of a circular cylinder in a circular polynya against wave number $k_{0}$. (As in Fig. 3.)

\subsection{Application: hydrodynamic loads on a FPSO floating in a polynya}

As shown in Fig. $5(a)$ and $(b)$, a floating structure FPSO is chosen for the case study. Its half-length $l$ is chosen as the characteristic length for non-dimensionalisation. The cross section of middle ship is rectangle, with its length and width respectively to be 1.5 and 0.5 . The bow and stern have the same shape, a half cylinder with radius as 0.25 . The draught of the FPSO is 0.3. On the mean wetted body surface of FPSO, $N_{B}=1958$ quadrilateral panels are used, which is sufficient for convergence. The rotational centre is assumed at the geometry centre of FPSO. The water depth and ice sheet thickness are taken as $H=10$ and $h=0.1$ respectively. Five cases will be considered in this section, i.e. 1) case 1 is for a circular polynya with its radius as $c=3$, and the FPSO is centrally located;2) case 2 is similar to case 1 , but with the shape of polynya as a square with its half-length taken to be $d=3 ; 3$ ) case 3 is similar to case 1 , but with a much larger polynya or $c=5 ; 4)$ case 4 is similar to case 3 but with geometry centre at $(1,0,-0.15) ; 5)$ case 5 is similar to case 3 but with geometry centre at $(0,1,-0.15)$. Without losing generality, in all of these five cases the incident flexural-gravity wave angle is taken as $\beta=\pi / 4$. The wave number $k_{0}$ varies from 0.01 to 3.0 , and the increment has been chosen to be sufficiently small, or 0.01 , to capture the position and the value of the peak of the curve.

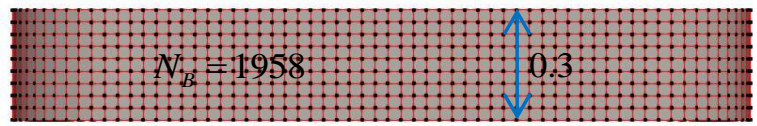

(a)

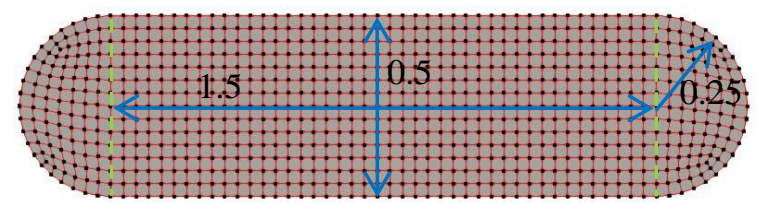

(b)

Fig. 5. The geometry shape and mesh on the mean wetted body surface of FPSO. 


\subsubsection{Hydrodynamic loads on a FPSO floating in polynya with different shapes}

Here, case 1 and case 2 respectively for circular and square polynyas will be considered to show the effects of polynya shape on the hydrodynamic loads. It may be noticed that the main dimensions of both cases are the same or $c=d=3$. In latter case, the four corners of the square polynya are smoothed by circular arc of radius 1.5. As shown in Fig. $6(a)$ and (b), the circular and square ice sheet edges are respectively discretized into $N_{\Gamma}=100$ and $N_{\Gamma}=120$ straight line segments, and convergent results can be obtained.

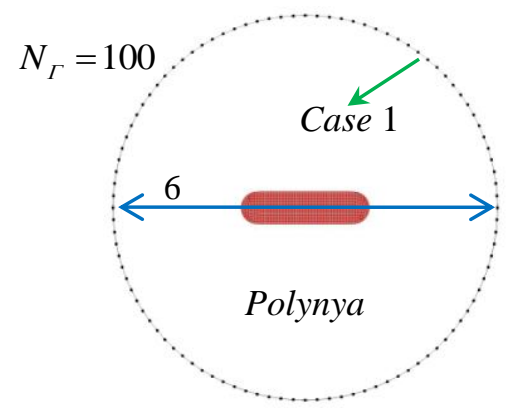

(a)

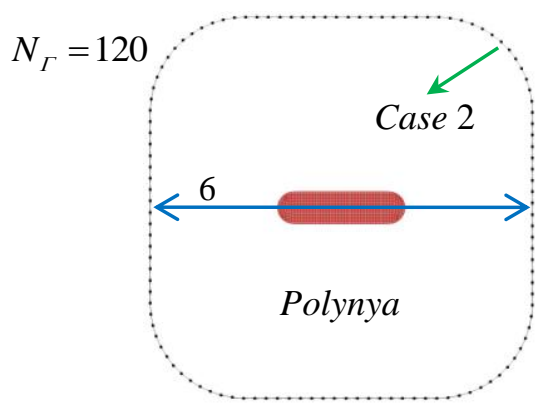

(b)

Fig. 6. The mesh on the ice sheet edge. (a) case 1 for a circular polynya $\left(N_{\Gamma}=100\right)$; $(b)$ case 2 for a square polynya with smoothed corners $\left(N_{\Gamma}=120\right)$.

The computed diagonal terms of the added mass and damping coefficient are shown in Fig. 7 against wave number $k_{0}$, while the wave exciting force are plotted in Fig. 8. To see the asymptotic behaviours as $h \rightarrow 0$, the numerical results for a very small ice thickness or $h=0.001$ are plotted, too. As a comparison, the results corresponding to the open sea or $h=0$ are also provided. It should be mentioned that for $h=0$ both the hybrid method and the direct method have been used, and graphically indistinguishable results are obtained. Here, direct method means that the velocity potential are computed through the boundary integral equation in (21) over the body surface only. From these two figures, it can be seen that when $h$ is small, the numerical results are very close to those for open water. In fact, as $h \rightarrow 0$ both the dispersion equation and the eigenfunctions for an ice sheet will tend to those for open water, and the inner product in equation (43) will become the usual definition of orthogonality. Therefore, as $h \rightarrow 0$ the solution for sub-domain $\Omega_{2}$ will serve as a radiation condition of free surface for sub-domain $\Omega_{1}$.

When the wave number is small, the results of the hydrodynamic loads for polynya are very close to those for open sea, as can be observed from Figs. 7 and 8. From equations (5) and (6), we have that when $k_{0} \rightarrow 0$ or $\omega \rightarrow 0$, both the leading terms of the boundary conditions on free surface and ice sheet will tend to be the same or $\partial \phi_{i} / \partial z=0$. This means that the upper surface boundary condition for polynya will be the same as that for open sea as $k_{0} \rightarrow 0$. Thus the results 
for FPSO floating in polynya will be very similar to those for open sea when $k_{0}$ is small.

However, when $k_{0}$ becomes larger, discrepancy appears. The hydrodynamic loads for polynya exhibit an oscillatory behavior around those for open sea, as shown in Figs. 7 and 8. From the dispersion equations (15) and (17), we have that when waves propagate from open sea to the ice or the other way around, there will be a change of the dispersion relation, i.e. the waves will be both reflected back to the original region and transmitted to the other region. Thus, as the wave generated or disturbed by the structure encounters the ice edge, part of it will be reflected back to the structure, and then will be further reflected back to the ice edge. This resembles the "sloshing wave in a tank", leading to the oscillations of the hydrodynamic loads. These oscillatory features are similar to those in a two dimensional polynya [40]. An important difference is that for three dimensional polynya, the wave energies will also propagate laterally. This means that the hydrodynamic loads on the body surface will be affected not only by the dimension of polynya, but also by its geometry. This can be clearly seen in Figs. 7 and 8 that when the wave number $k_{0}$ is not small, the hydrodynamic loads for a square polynya will exhibit a quite different oscillatory patterns with those for a circular polynya, although the main dimension of these two polynyas are similar. Specifically, for the former case, in addition to the overall variations, there are also some local oscillations vary sharply from peaks to the troughs. These are similar to the resonant motions of fluid in a tank, in which circular and rectangular shapes would resemble different natural frequencies [2]. Another similar problem is a ship floating in a harbor, and very large hydrodynamic loads on the structure could occur at some specific wave numbers [36]. However, a significant physical difference is that in our work only part of wave energy will be reflected back due to the change of wave dispersion relation, while in the former two cases the wave energy will be fully reflected by the solid wall due to the non-penetration condition. All these show the importance of the effects of polynya shape on the hydrodynamic loads for a realistic structure operating in icy waters.

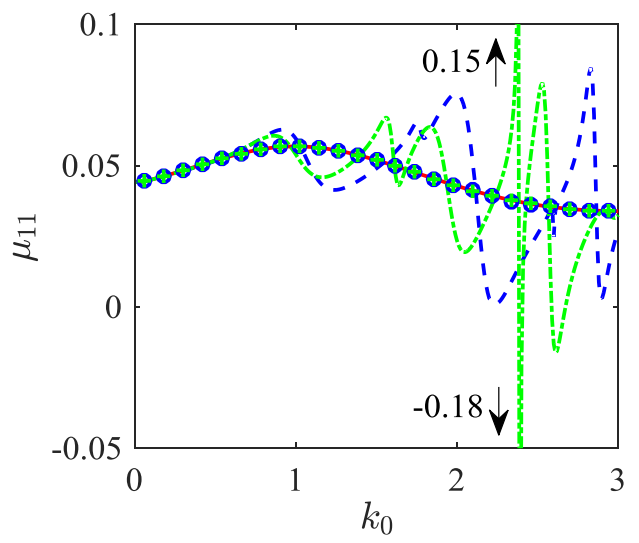

(a)

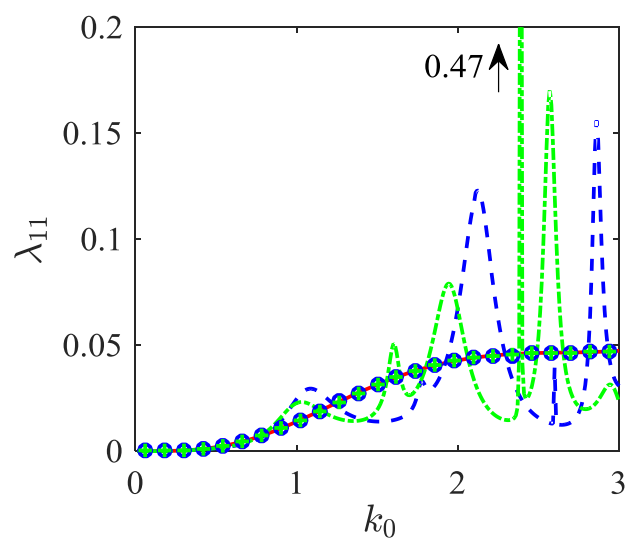

(b) 


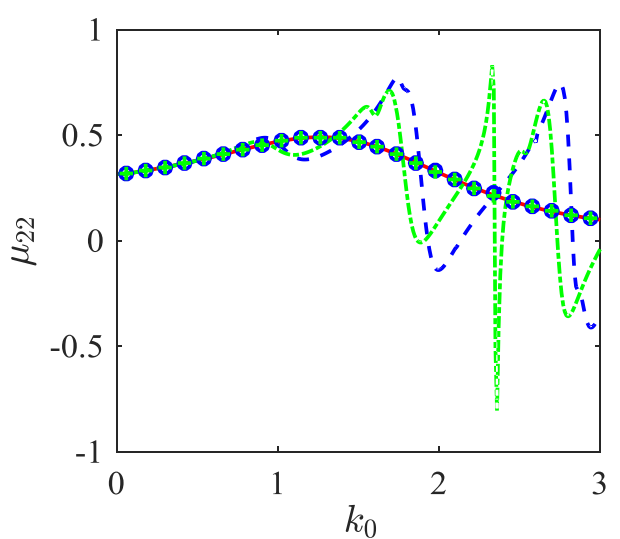

(c)

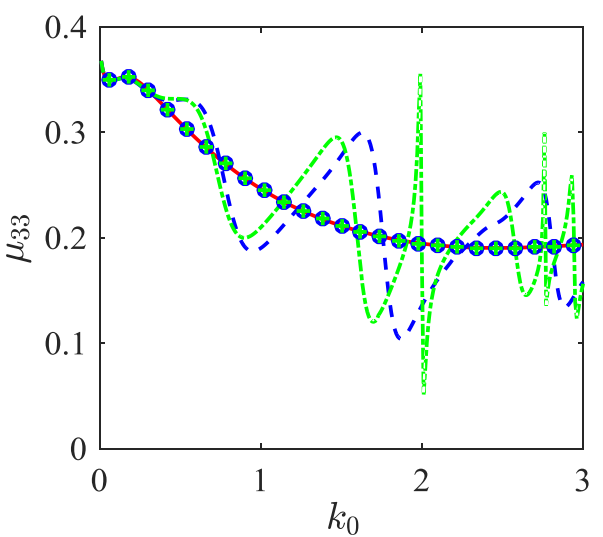

(e)

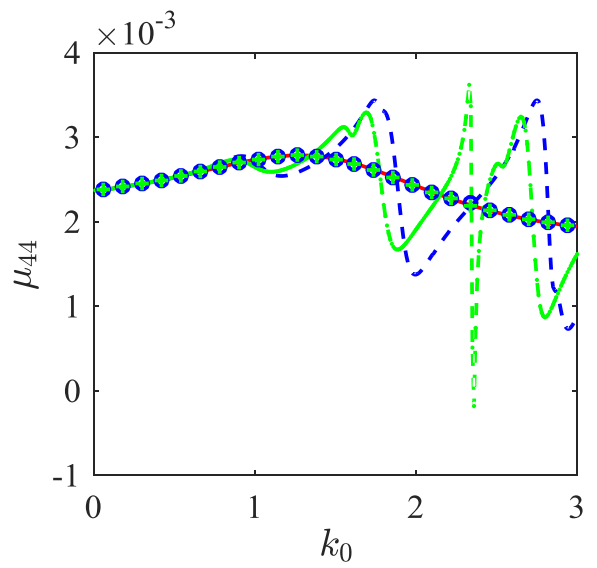

(g)

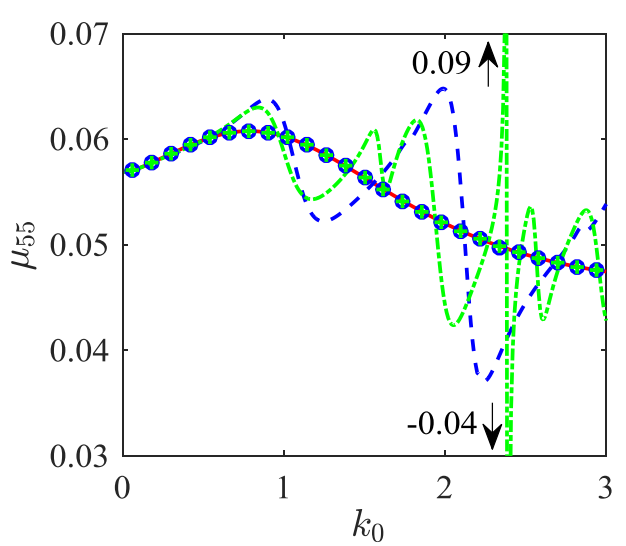

(i)

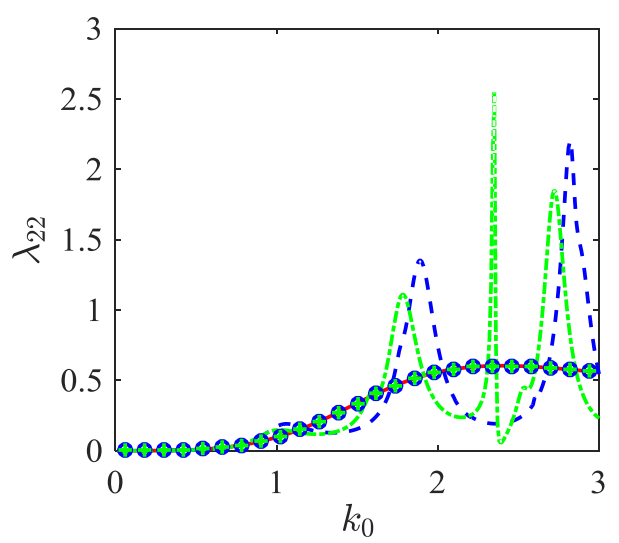

(d)

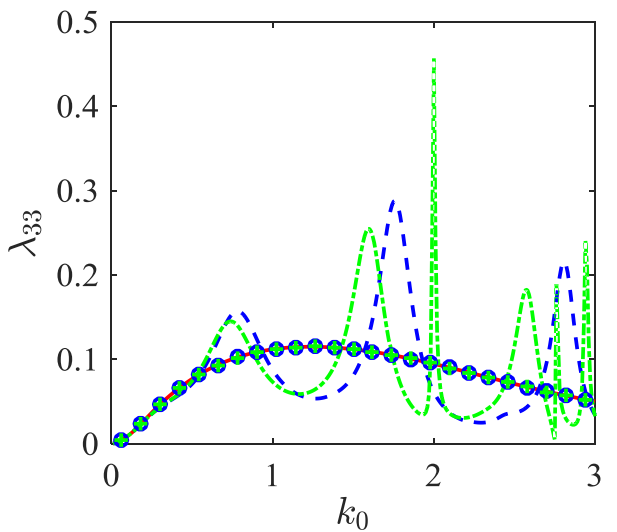

(f)

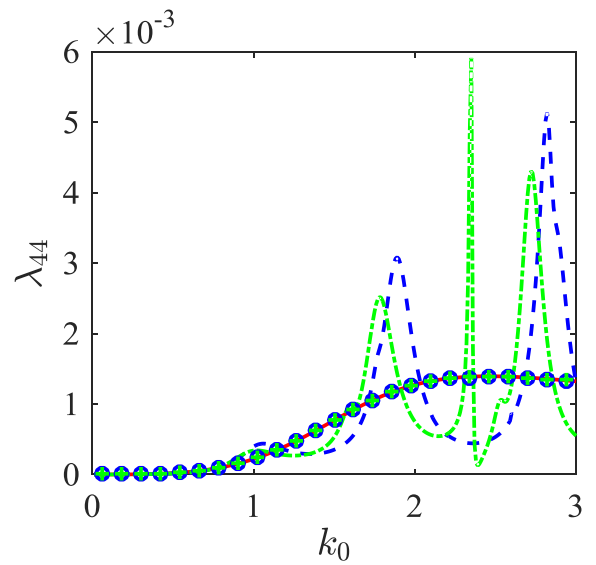

(h)

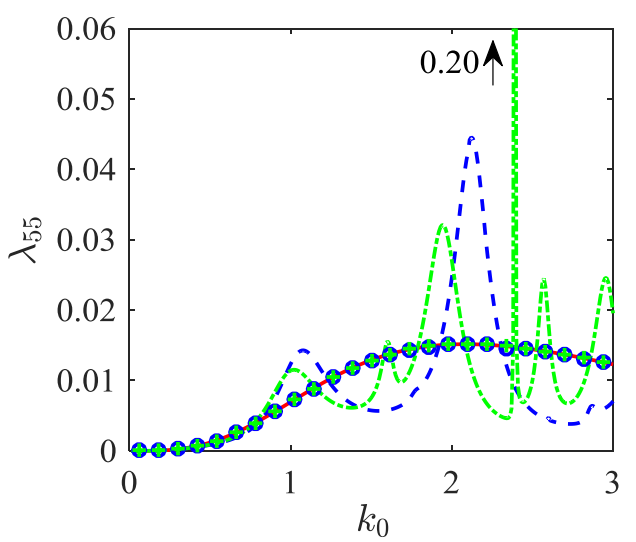

(j) 


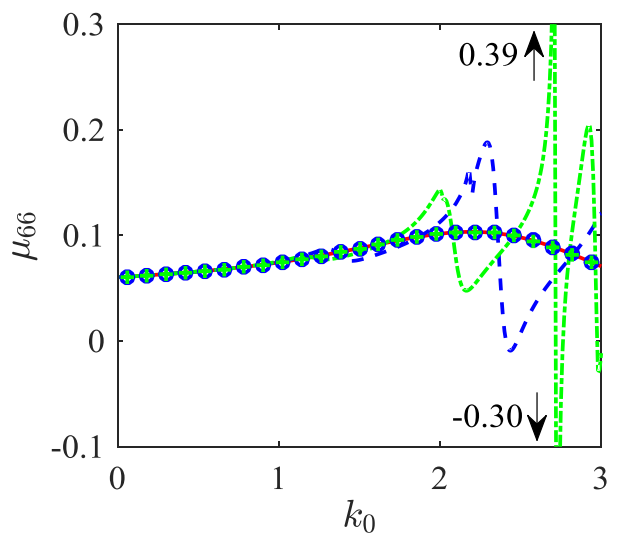

(k)

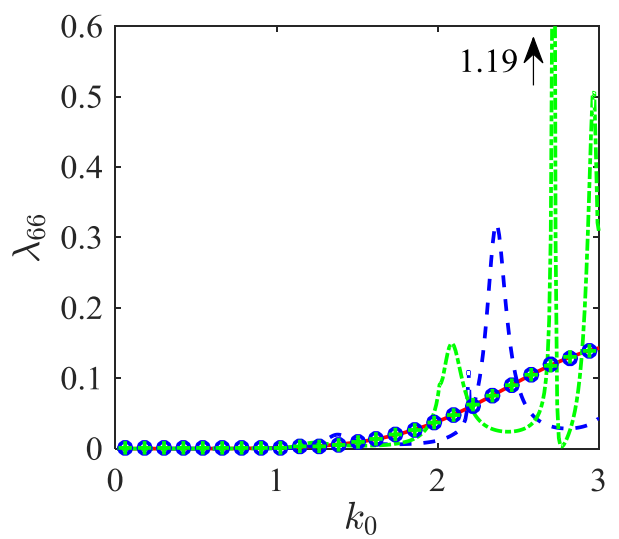

(l)
1

2

3

4

5

Fig. 7. Added mass $\mu_{j k}$ and damping coefficient $\lambda_{j k}$ of a FPSO floating in polynya with different shapes against wave number $k_{0}$. Solid lines: results for open sea; dashed lines: results for circular polynya; dash-dotted lines: results for square polynya; circles: similar to dashed lines but for $h=0.001$; pluses: similar to dash-dotted lines but for $h=0.001 .(l=1, c=d=3, H=10, h=0.1, m=0.09, L=4.5582, \beta=\pi / 4)$

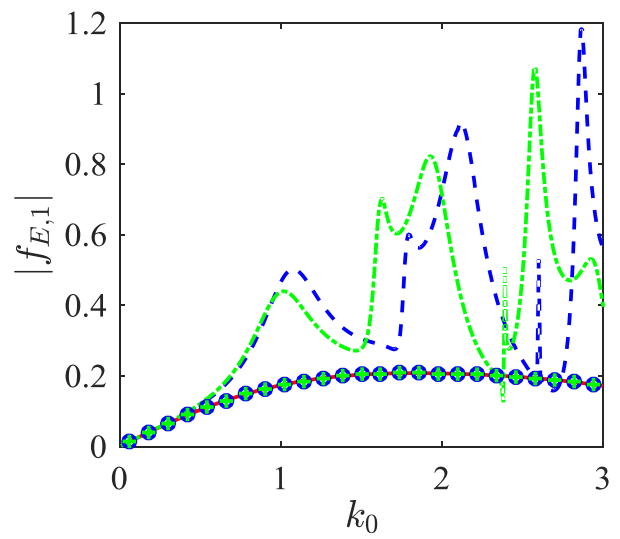

(a)

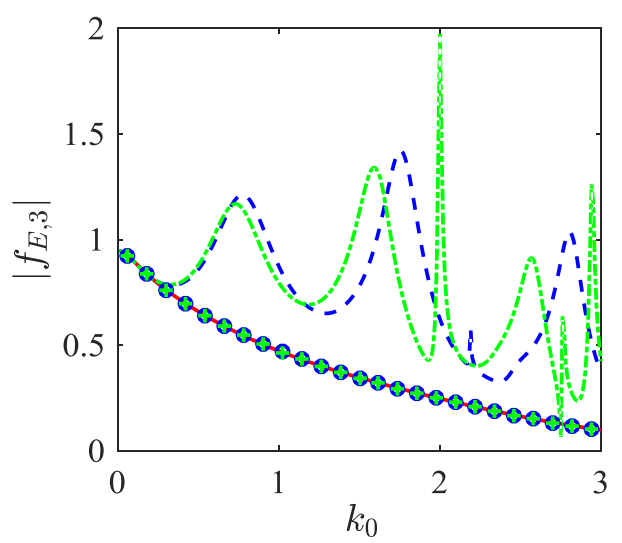

(c)

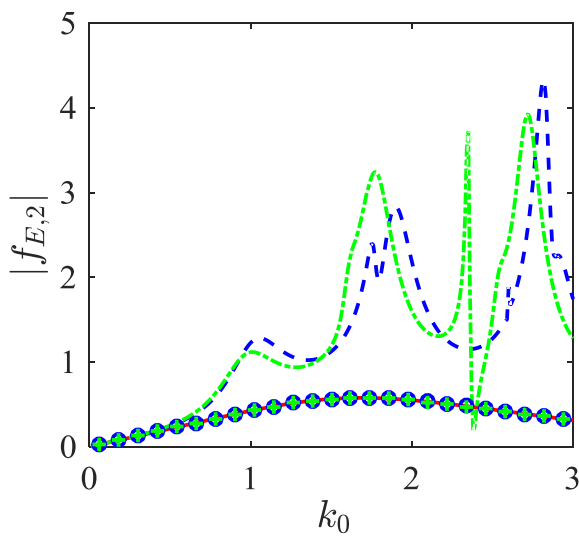

(b)

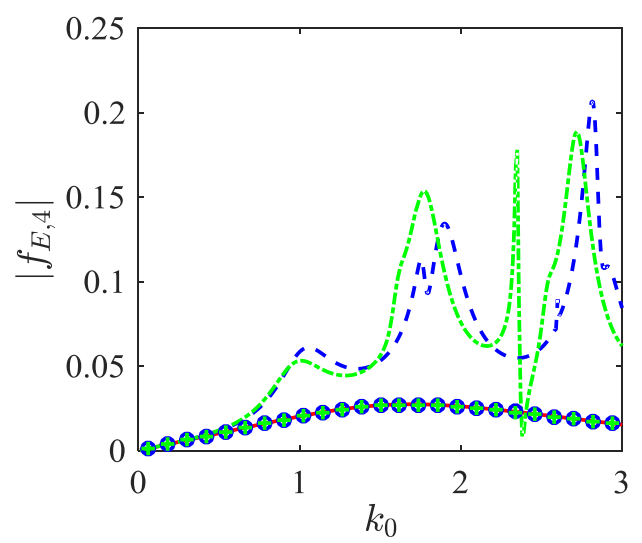

(d) 


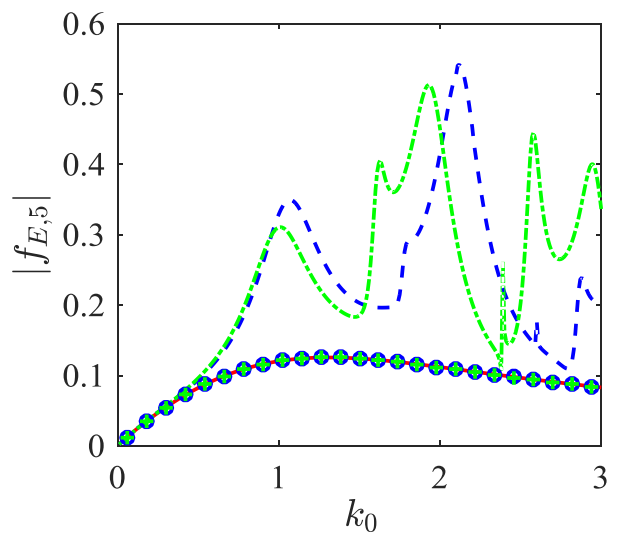

(e)

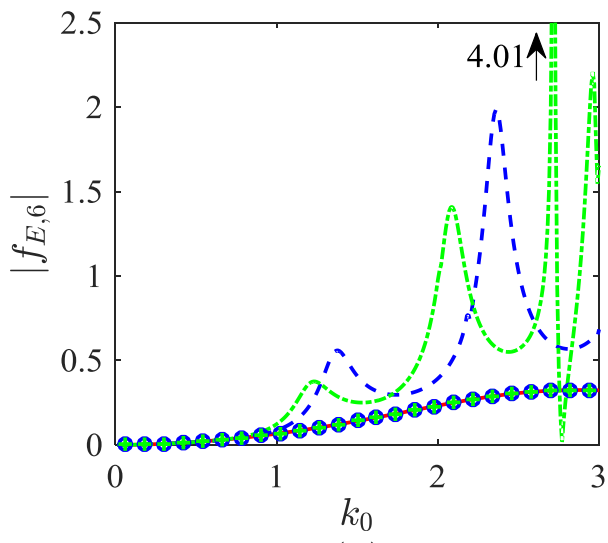

$(f)$

Fig. 8. Wave exciting force $f_{E, j}$ of a FPSO floating in polynya with different shapes against wave number $k_{0}$. (As in Fig. 7.)

\subsubsection{Hydrodynamic loads on a FPSO floating in polynya with different positions}

Computations are now carried out for cases 3 to 5 to show the effects of floating position of FPSO in polynya on the hydrodynamic loads. In these three cases, the polynya has the same shape as that in Fig. 6 (a), but with a much larger polynya radius or $c=5$. Here, three different floating positions of FPSO are considered, i.e. centrally located, near right ice sheet edge with geometry centre at $(1,0,-0.15)$, near top ice sheet edge with geometry centre at $\vec{r}_{0}=(0,1,-0.15)$, as shown in Fig. 9.

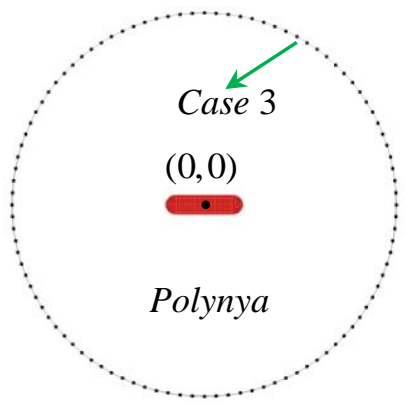

(a)

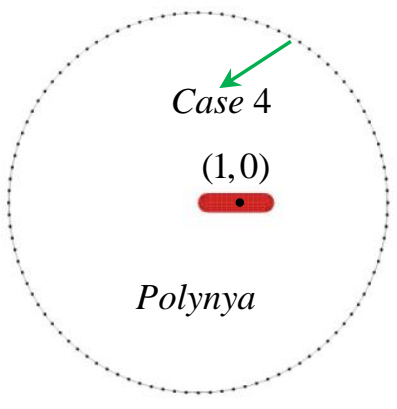

(b)

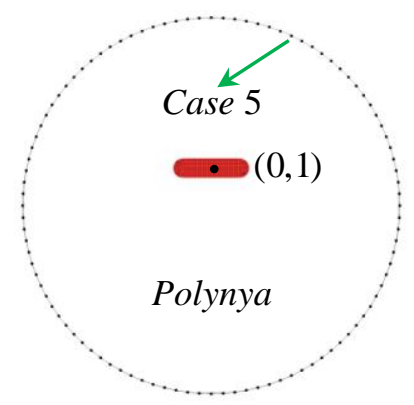

(c)

Fig. 9. The floating position of FPSO in a circular polynya with radius $c=5$. (a) case 3 for centrally located; $(b)$ case 4 for near the right ice sheet edge; $(c)$ case 5 for near the top ice sheet edge.

Fig. 10 shows the diagonal terms of the hydrodynamic coefficients of a FPSO floating in a circular polynya in different positions, i.e. centrally located, near the right and top ice sheet edges respectively. The corresponding wave exciting force is given in Fig. 11. For the centrally located case, it differs from that in Figs. 7 and 8 only by the size of the polynya. As one can expect, the oscillatory behaviour of the results will be very much affected by $k_{0} c$. Thus there are more peaks and troughs in the Figs. 10 and 11 with larger $\mathrm{c}$ within the same wave number span as in Figs. 7 and 8 . When the polynya shape keeps the same and floating position of the body varies, the results 
in Figs. 10 and 11 reveal that the hydrodynamic loads can be much more oscillatory than those for centrally located case. These may be partly explained by the approximate formulations in $\mathrm{Li}$, Shi and $\mathrm{Wu}$ [40] for a 2D body floating in a wide polynya. In such a simplified model, through ignoring the evanescent wave modes in the coupled ice body interactions, the hydrodynamic loads on the body in polynya can be computed via the solutions of wave ice interactions without the body and wave body interactions without the ice. The explicit equations show that at a given wave number, the hydrodynamic loads on the body will oscillate periodically against two spatial variables, i.e. the width of the polynya and the distance between the polynya centre and body centre. When the value of these two parameters increase, the corresponding oscillatory period will decrease or the results will be more oscillatory. While for a centrally located body, only the first oscillatory term will appear because of symmetry. However, when the body is away from the polynya centre, there will be two oscillatory terms, leading to a much more oscillatory results.

The wave elevation $w_{0}$ in polynya along the ice edge due to incident and diffracted waves is shown in Fig. 12 against $\theta=\arctan (y / x)$, at different wave numbers $k_{0}$. While in Fig. 13, $w_{0}$ is provided against $k_{0}$ at different ice edge positions $\theta$. From these two figures we observe that when $k_{0} \rightarrow 0$ or the wave length tends to infinity, $w_{0}$ will tend to 1 or the diffracted wave will be zero. This is consistent with the fact that as $k_{0} \rightarrow 0$ both the boundary conditions on ice sheet and free surface will tend to be the same, i.e. no diffracted wave will be generated by the polynya and then by the body due to an incident wave of infinite wavelength. As $k_{0}$ increases, it can be seen that the variation of $w_{0}$ against $\theta$ for a given $k_{0}$ or against $k_{0}$ for a given $\theta$ becomes highly complex. Specifically, the wave elevation in polynya can be many times of the incident wave amplitude. Strictly speaking, the linear theory is for infinitesimal wave. In practical problems, this applicability depends on the accuracy desired. It is then possible that the linear theory may be valid for the incident wave, but the nonlinearity may become important in the polynya. In such a case, nonlinear correction should be introduced into the model, or the linear theory is valid only for even smaller incoming wave amplitude.

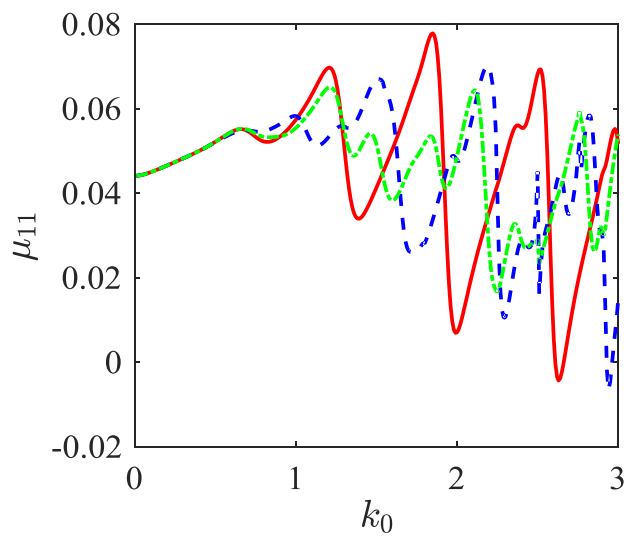

$(a)$

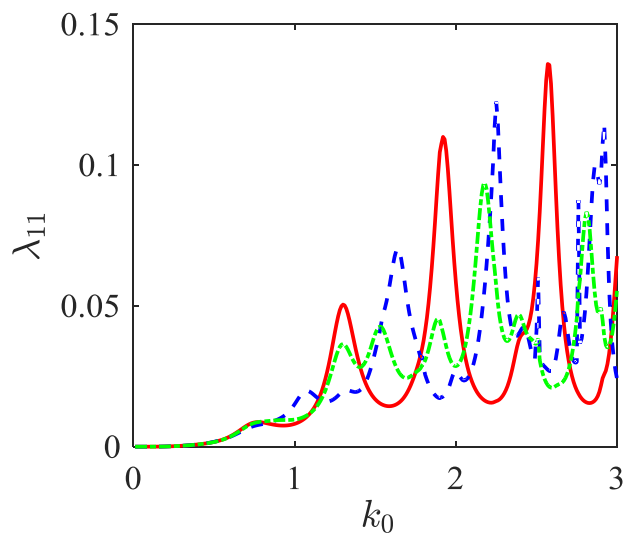

(b) 


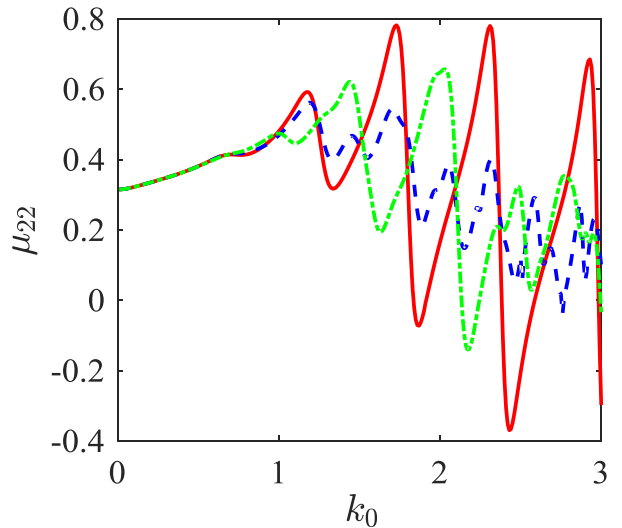

(c)

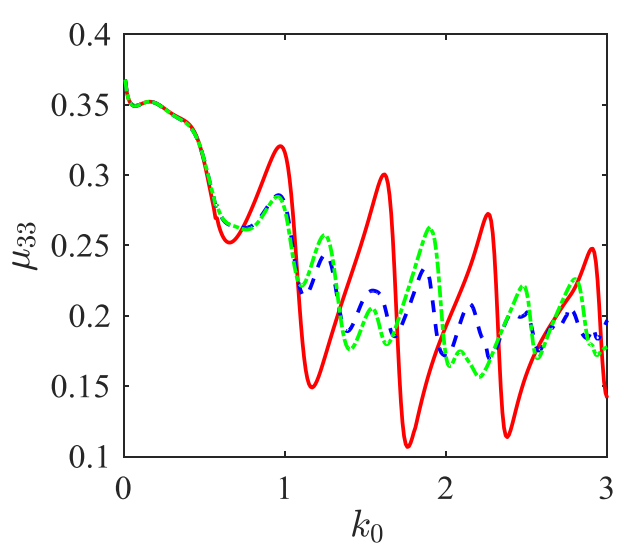

(e)

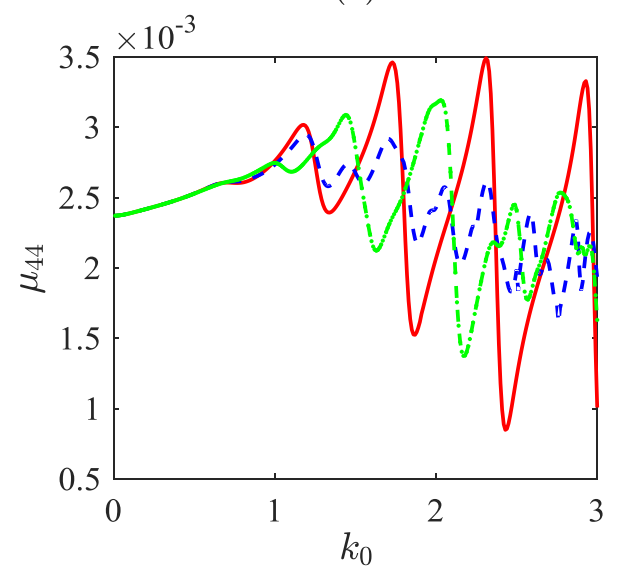

(g)

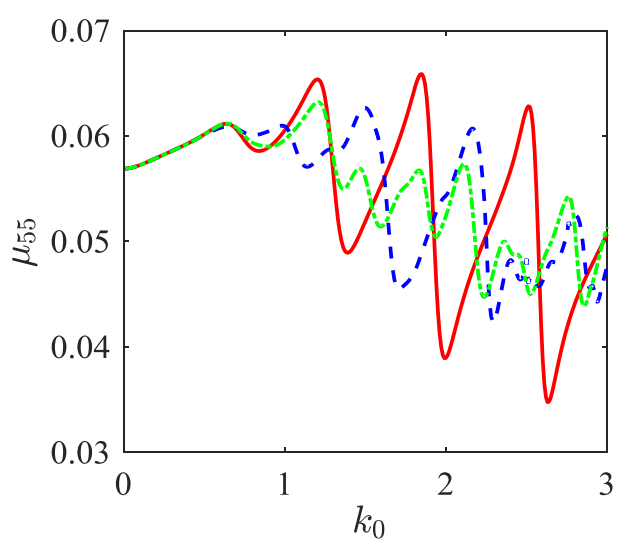

(i)

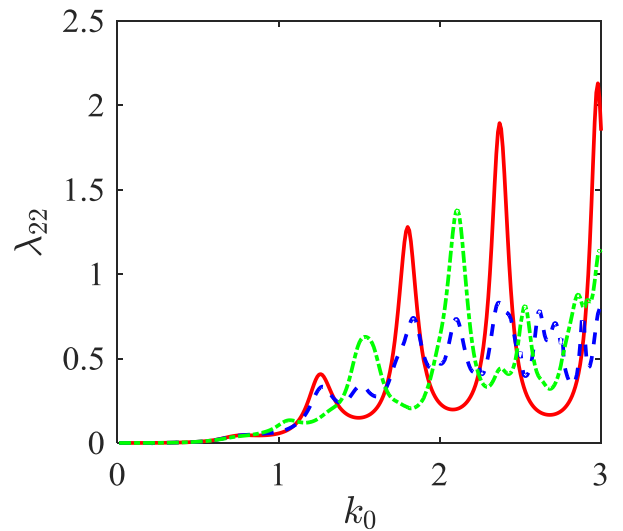

(d)

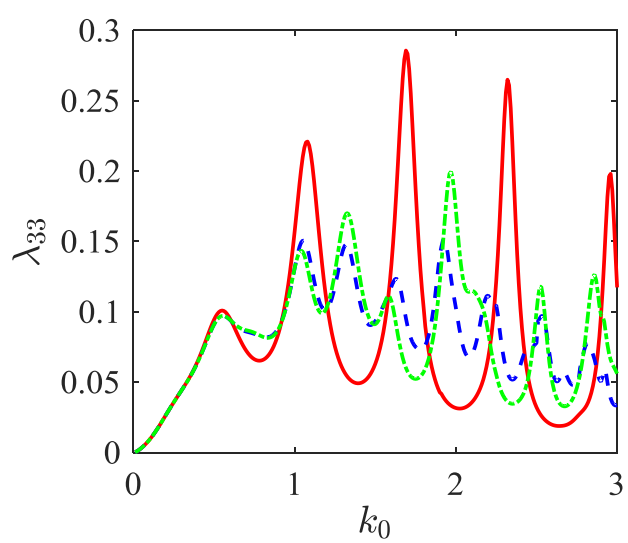

(f)

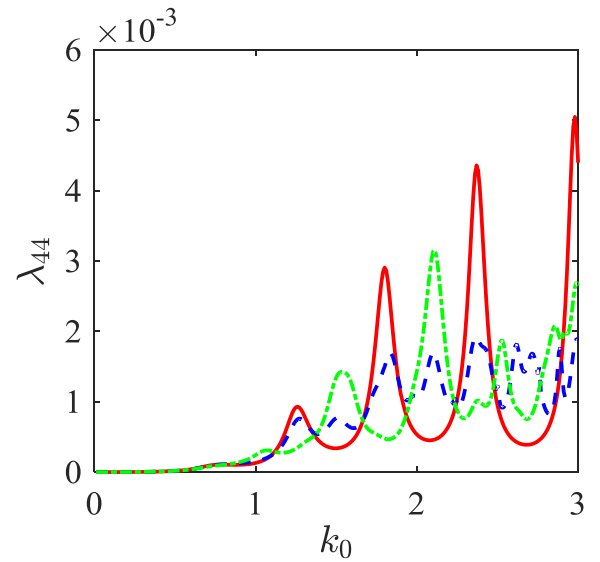

(h)

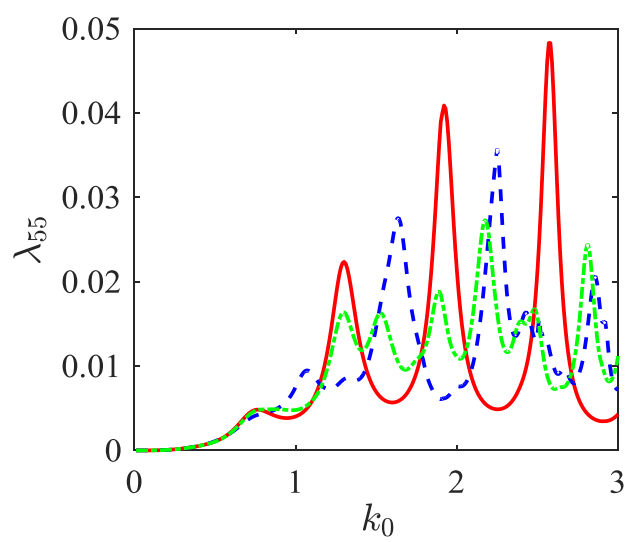

(j) 


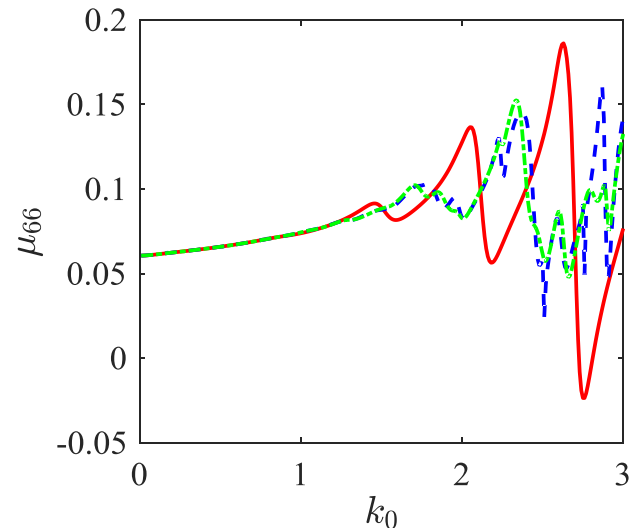

(k)

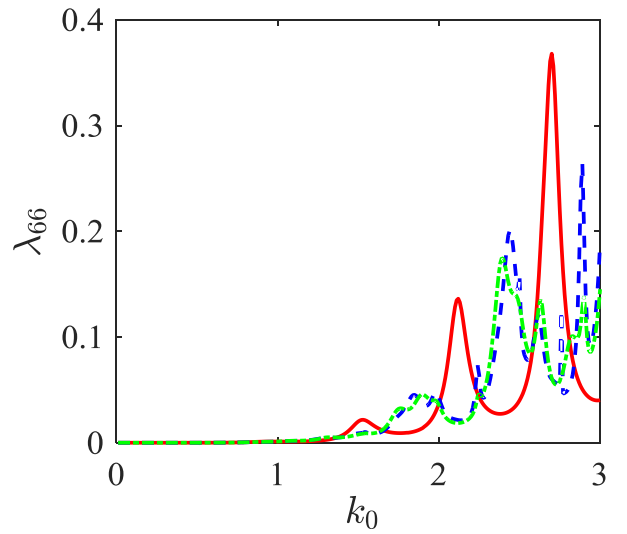

(l)
1

2

3

4

5

Fig. 10. Added mass $\mu_{j k}$ and damping coefficient $\lambda_{j k}$ of a FPSO floating in polynya with different positions against wave number $k_{0}$. Solid lines: centrally located with $\left(x_{0}, y_{0}\right)=(0,0)$; dashed lines: near the right ice sheet edge with $\left(x_{0}, y_{0}\right)=(1,0)$; dash-dotted lines: near the top ice sheet edge with $\left(x_{0}, y_{0}\right)=(0,1) .(l=1, c=5$, $H=10, h=0.1, m=0.09, L=4.5582, \beta=\pi / 4)$

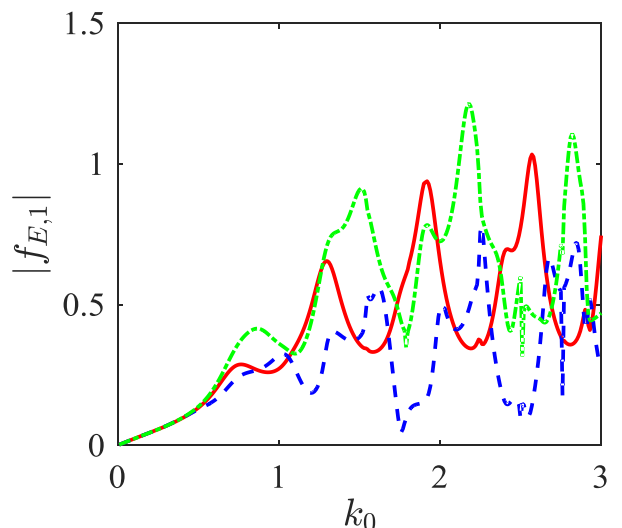

(a)

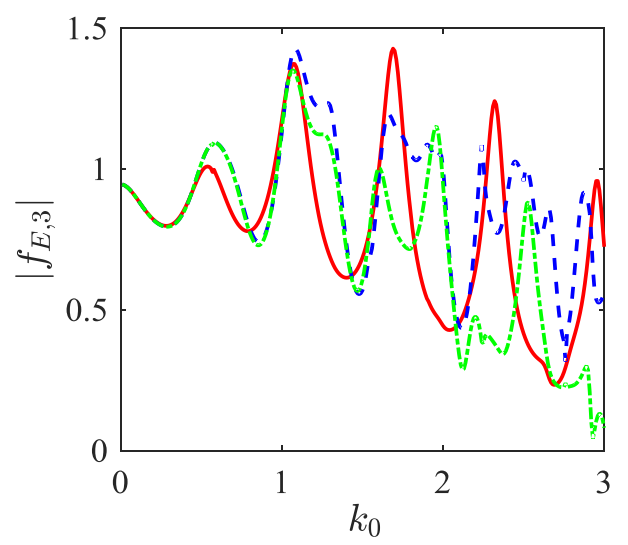

(c)

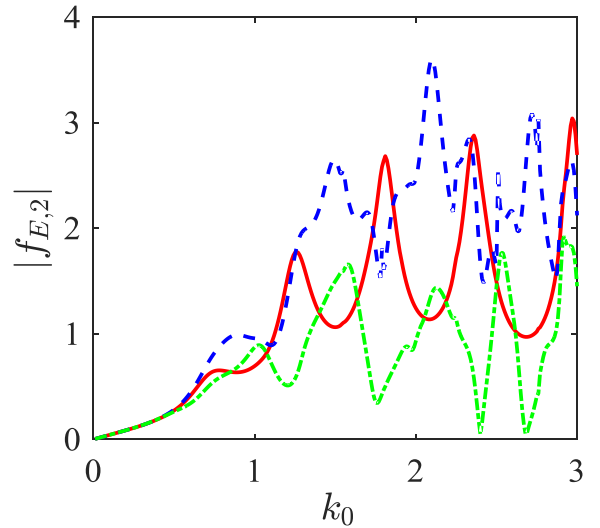

(b)

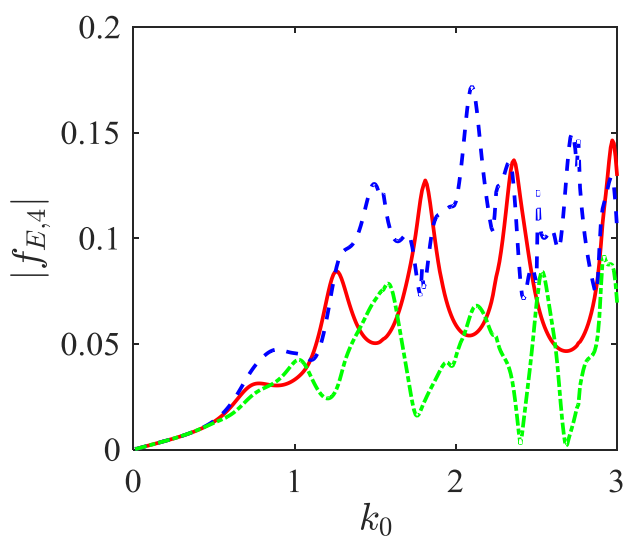

(d) 


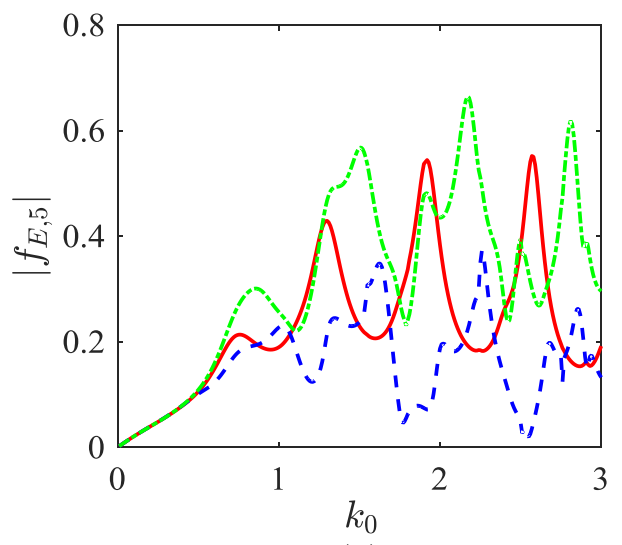

(e)

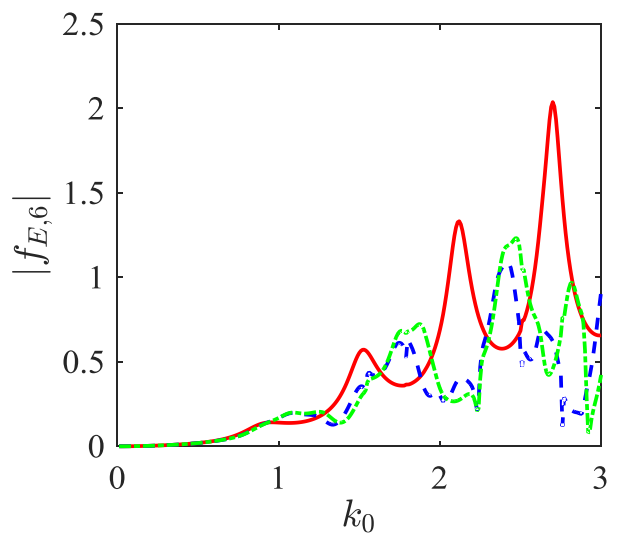

$(f)$
1

2

3

4

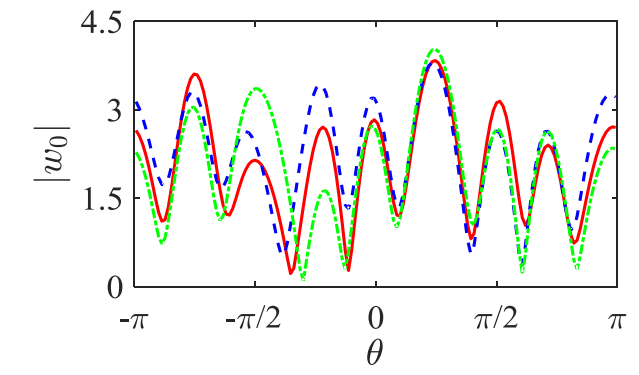

(c)

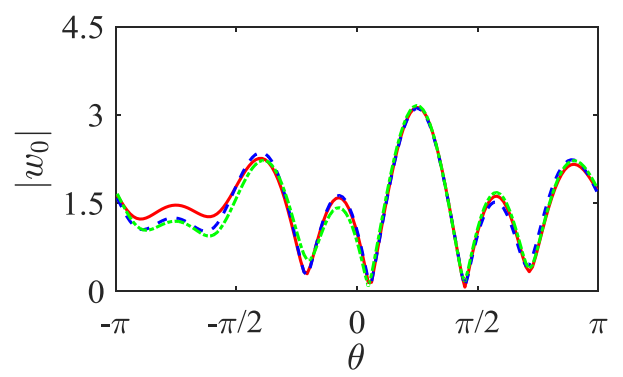

(b)

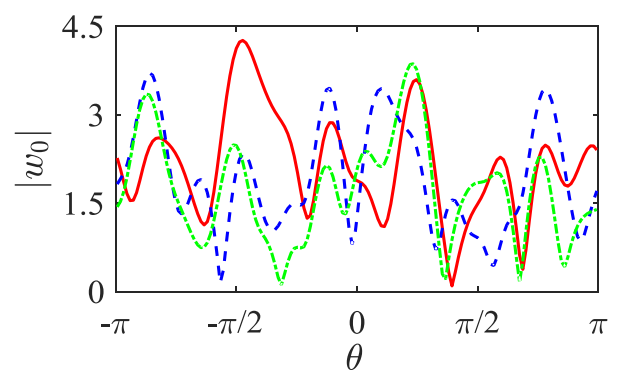

(d)
6

7

8

9

Fig. 12. Wave elevation $w_{0}$ in polynya along the ice edge due to incident and diffracted waves at different wave number $k_{0}$. (a) $k_{0}=0.01 ;$ (b) $k_{0}=1.0 ;$ (c) $k_{0}=2.0 ;$ (d) $k_{0}=3.0$. Solid lines: centrally located with $\left(x_{0}, y_{0}\right)=(0,0)$; dashed lines: near the right ice sheet edge with $\left(x_{0}, y_{0}\right)=(1,0)$; dash-dotted lines: near the top ice sheet edge with $\left(x_{0}, y_{0}\right)=(0,1) .(l=1, c=5, H=10, h=0.1, m=0.09, L=4.5582, \beta=\pi / 4)$

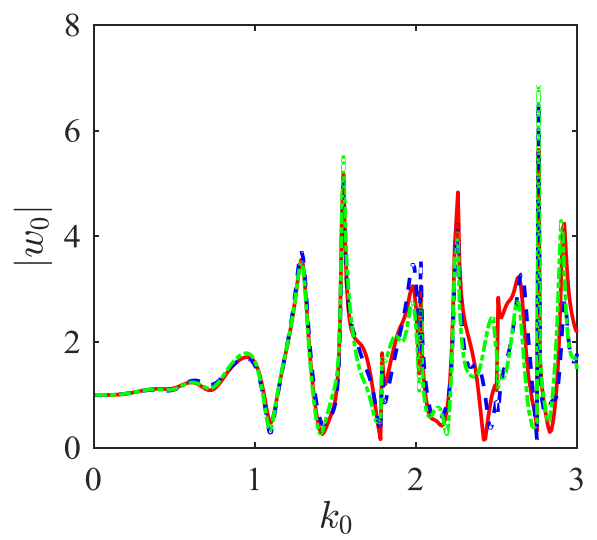

(a)

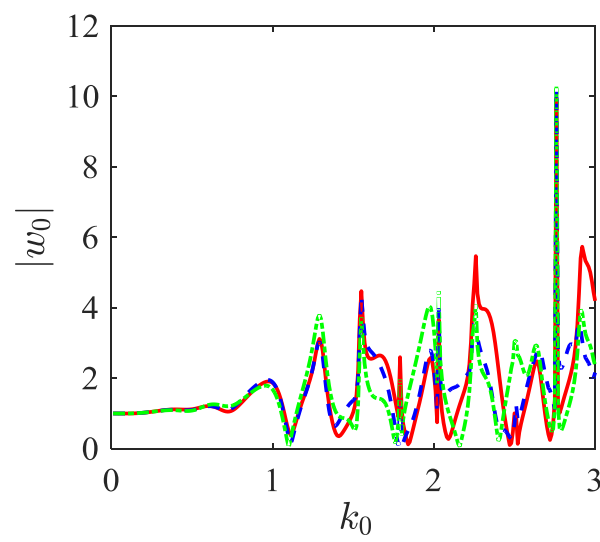

(b) 


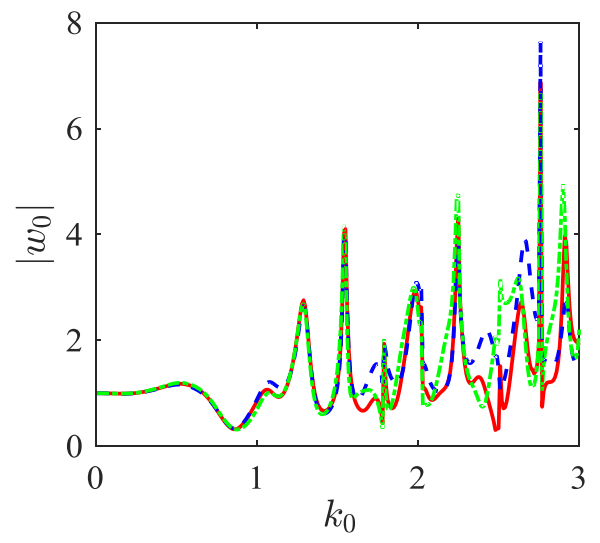

(c)

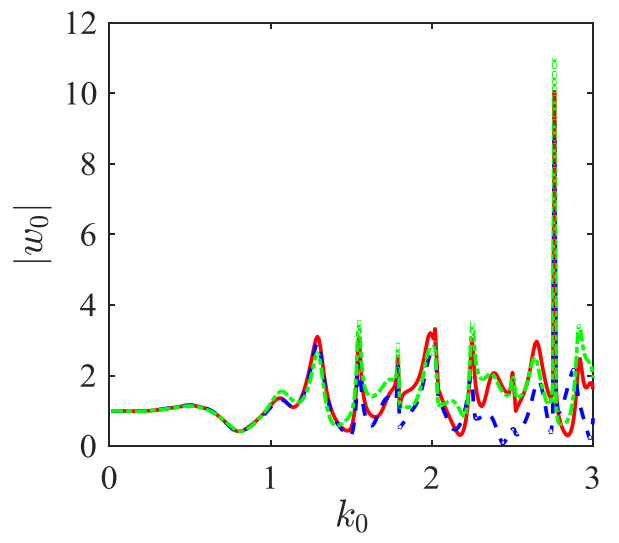

(d)

Fig. 13. Wave elevation $w_{0}$ in polynya due to incident and diffracted waves against wave number $k_{0}$ at different ice edge positions. (a) $\theta=-3.11$; (b) $\theta=-1.54$; (c) $\theta=0.03$; (d) $\theta=1.60$. Solid lines: centrally located with $\left(x_{0}, y_{0}\right)=(0,0)$; dashed lines: near the right ice sheet edge with $\left(x_{0}, y_{0}\right)=(1,0)$; dash-dotted lines: near the top ice sheet edge with $\left(x_{0}, y_{0}\right)=(0,1) .(l=1, c=5, H=10, h=0.1, m=0.09, L=4.5582$, $\beta=\pi / 4$ )

\section{Conclusions}

We have developed an accurate and efficient numerical solution approach for wave radiation and diffraction problems of a three dimensional floating structure in a polynya. To solve the governing equation with nonhomogeneous upper surface conditions, a vertical control surface along the ice edge is introduced, which separates the fluid into two sub-domains. In exterior region with an infinitely extended ice sheet, the upper surface condition contains up to fifth derivative of the potential. To satisfy this and the radiation condition analytically, the velocity potential has been written in the form of vertical eigenfunction series with the unknown functions in the horizontal direction, which satisfy the two dimensional Helmholtz equation and are then expressed in terms of the integral equation along the ice sheet edge. In interior region with a free surface, the velocity potential has been written in form of the integral equation over the structure and control surfaces, where the free surface Green function is taken as the kernel. On the control surface extended vertically from the ice edge, both the velocity potential and the Green function are expanded into the orthogonal eigen function series in the vertical direction. Thus, the integral over the control surface is transformed into the integral along the ice edge, the same as that in exterior domain. The unknowns in both interior and exterior sub-domains are solved together through an inner product, where the continuity condition of pressure and velocity is used. Through this the ice edge conditions are also satisfied.

Simulations have been first carried out for a bottom mounted vertical circular cylinder in a circular polynya, and comparison has been made with the analytical solution derived previously. Good agreements have been found, indicating that the method is accurate. The applicability of the 
method to a realistic offshore structure with complicated geometry is demonstrated, through applying the code WISPICE to the wave interactions with a FPSO floating in a polynya with circular and square shapes respectively. The method can be used and extended to study the more complex physical problems, for example, multiple body or multiple polynya problem by distributing panels over each body surface or dividing the fluid domain into multiple sub-domains. The same matching procedure can be followed. The method can be also easily extended to polynya surrounded by an ice sheet of finite extent. In this case, in the third domain beyond the ice sheet, the ice sheet thickness can be taken as zero and the same method used in the domain below the ice sheet can be followed. Compared with the other methods for this type of linearized problem, such as finite difference method or the finite element method, in the present hybrid method, only the body surface and ice sheet edge need to be discretized, and the boundary conditions on free surface and ice sheet as well as the radiation condition are all satisfied automatically through either the Green function or the vertical modes of the potential. However, the method is limited to the scope of the linear velocity potential theory, which is not valid when the wave amplitude or the body motion amplitude is large compared with the wavelength or the body dimension.

\section{Acknowledgement}

This work is supported by Lloyd's Register Foundation, to which the authors are most grateful. Lloyd's Register Foundation helps to protect life and property by supporting engineering-related education, public engagement, and the application of research. This work is also supported by the National Natural Science Foundation of China (Grant No. 51709131 and 51879123 )

\section{References}

[1] F. Smith, A. Korobkin, E. Parau, D. Feltham, V. Squire, Modelling of sea-ice phenomena, Philosophical Transactions of the Royal Society, A376 (2018) 20180157.

[2] C.M. Linton, P. Mciver, Handbook of mathematical techniques for wave/structure interactions, CRC Press, United States, 2001.

[3] R.W. Yeung, Numerical methods in free-surface flows, Annual Review of Fluid Mechanics, 14 (1982) 395-442.

[4] C.C. Mei, M. Stiassnie, K.P. Yue, Theory and applications of ocean surface waves part 1: linear aspects, World Scientific Publishing, Singapore, 2005.

[5] H.D. Maniar, J.N. Newman, Wave diffraction by a long array of cylinders, Journal of Fluid Mechanics, 339 (1997) 309-330.

[6] J.N. Newman, Trapped-wave modes of bodies in channels, Journal of Fluid Mechanics, 812 (2017) 178-198. 
[7] H. Liang, X.B. Chen, A new multi-domain method based on an analytical control surface for linear and second-order mean drift wave loads on floating bodies, Journal of Computational Physics, 347 (2017) 506-532.

[8] V.A. Squire, J.P. Dugan, P. Wadhams, P.J. Rottier, A.K. Liu, Of ocean waves and sea ice, Annual Review of Fluid Mechanics, 27 (1995) 115-168.

[9] V.A. Squire, Of ocean waves and sea-ice revisited, Cold Regions Science and Technology, 49 (2007) 110-133.

[10] V.A. Squire, Past, present and impendent hydroelastic challenges in the polar and subpolar seas, Philosophical Transactions of the Royal Society, A369 (2011) 2813-2831.

[11] G.X. Wu, A numerical scheme for calculating the mj terms in wave-current-body interaction problem, Applied Ocean Research, 13 (1991) 317-319.

[12] G.X. Wu, R. Eatock Taylor, Hydrodynamic forces on submerged oscillating cylinders at forward speed, Proceedings of the Royal Society of London, A414 (1987) 149-170.

[13] Z.F. Li, Y.Y. Shi, G.X. Wu, Interaction of waves with a body floating on polynya between two semi-infinite ice sheets, Journal of Fluids and Structures, 78 (2018) 86-108.

[14] G.D. Dean, Green's functions with applications, CRC Press, United States, 2001.

[15] T. Sahoo, T.L. Yip, A.T. Chwang, Scattering of surface waves by a semi-infinite floating elastic plate, Physics of Fluids, 13 (2001) 3215-3222.

[16] C. Fox, V.A. Squire, Reflection and transmission characteristics at the edge of shore fast sea ice, Journal of Geophysical Research Oceans, 95 (1990) 11629-11639.

[17] C. Fox, V.A. Squire, On the oblique reflexion and transmission of ocean waves at shore fast sea ice, Philosophical Transactions of the Royal Society, A347 (1994) 185-218.

[18] N.J. Balmforth, R.V. Craster, Ocean waves and ice sheets, Journal of Fluid Mechanics, 395 (1999) 89-124.

[19] C.M. Linton, H. Chung, Reflection and transmission at the ocean/sea-ice boundary, Wave Motion, 38 (2003) 43-52.

[20] M.H. Meylan, V.A. Squire, Finite-floe wave reflection and transmission coefficients from a semi-infinite model, Journal of Geophysical Research, 98 (1993) 12537-12542.

[21] M.H. Meylan, V.A. Squire, The response of ice floes to ocean waves, Journal of Geophysical Research Atmospheres, 99 (1994) 891-900.

[22] H. Chung, C.M. Linton, Reflection and transmission of waves across a gap between two semi-infinite elastic plates on water, Quarterly Journal of Mechanics and Applied Mathematics, 58 (2005) 1-15.

[23] T.D. Williams, V.A. Squire, Scattering of flexural-gravity waves at the boundaries between three floating sheets with applications, Journal of Fluid Mechanics, 569 (2006) 113-140. 
[24] V.A. Squire, T.W. Dixon, How a region of cracked sea ice affects ice-coupled wave propagation, Annals of Glaciology, 33 (2001) 327-332.

[25] D.V. Evans, R. Porter, Wave scattering by narrow cracks in ice sheets floating on water of finite depth, Journal of Fluid Mechanics, 484 (2003) 143-165.

[26] R. Porter, D.V. Evans, Scattering of flexural waves by multiple narrow cracks in ice sheets floating on water, Wave Motion, 43 (2006) 425-443.

[27] K. Mattsson, E.M. Dunham, J. Werpers, Simulation of acoustic and flexural-gravity waves in ice-covered oceans, Journal of Computational Physics, 373 (2018) 230-252.

[28] M.H. Meylan, V.A. Squire, Response of a circular ice floe to ocean waves, Journal of Geophysical Research Oceans, 101 (1996) 8869-8884.

[29] F. Montiel, L.G. Bennetts, V.A. Squire, F. Bonnefoy, P. Ferrant, Hydroelastic response of floating elastic discs to regular waves. Part 2. Modal analysis, Journal of Fluid Mechanics, 723 (2013) 629-652.

[30] C.D. Wang, M.H. Meylan, A higher-order-coupled boundary element and finite element method for the wave forcing of a floating elastic plate, Journal of Fluids and Structures, 19 (2004) 557-572.

[31] L.G. Bennetts, T.D. Williams, Wave scattering by ice floes and polynyas of arbitrary shape, Journal of Fluid Mechanics, 662 (2010) 5-35.

[32] O.M. Faltinsen, Wave loads on offshore structures, Annual Review of Fluid Mechanics, 22 (1990) 35-56.

[33] B.Z. Zhou, G.X. Wu, Resonance of a tension leg platform exited by third-harmonic force in nonlinear regular waves, Philosophical Transactions of the Royal Society, A373 (2015) 21.

[34] Y.L. Shao, O.M. Faltinsen, A harmonic polynomial cell (HPC) method for 3D Laplace equation with application in marine hydrodynamics, Journal of Computational Physics, 274 (2014) 312-332.

[35] X.B. Chen, On the side wall effects upon bodies of arbitrary geometry in wave tanks, Applied Ocean Research, 16 (1994) 337-345.

[36] Y.Y. Shi, Z.F. Li, G.X. Wu, Motion of a floating body in a harbour by domain decomposition method, Applied Ocean Research, 78 (2018) 223-240.

[37] P. Kumar, H.W. Zhang, K.T. Kim, D.A. Yuen, Modeling wave and spectral characteristics of moored ship motion in Pohang New Harbor under the resonance conditions, Ocean Engineering, 119 (2016) 101-113.

[38] I.V. Sturova, Radiation of waves by a cylinder submerged in water with ice floe or polynya, Journal of Fluid Mechanics, 784 (2015) 373-395.

[39] K. Ren, G.X. Wu, G.A. Thomas, Wave excited motion of a body floating on water confined between two semi-infinite ice sheets, Physics of Fluids, 28 (2016) 127101. 
[40] Z.F. Li, Y.Y. Shi, G.X. Wu, Interaction of wave with a body floating on a wide polynya,

2 Physics of Fluids 29 (2017) 097104.

3 [41] I.V. Sturova, The effect of a crack in an ice sheet on the hydrodynamic characteristics of a 4 submerged oscillating cylinder, Journal of Applied Mathematics and Mechanics, 79 (2015) $5 \quad 170-178$.

6 [42] Z.F. Li, G.X. Wu, C.Y. Ji, Wave radiation and diffraction by a circular cylinder submerged 7 below an ice sheet with a crack, Journal of Fluid Mechanics, 845 (2018) 682-712.

8 [43] Z.F. Li, G.X. Wu, C.Y. Ji, Interaction of wave with a body submerged below an ice sheet with 9 multiple arbitrarily spaced cracks, Physics of Fluids 30 (2018) 057107.

[44] A.A. Korobkin, S. Malenica, T. Khabakhpasheva, Interaction of flexural-gravity waves in ice cover with vertical walls, Philosophical Transactions of the Royal Society, A376 (2018) 20170347.

[45] K. Ren, G.X. Wu, C.Y. Ji, Diffraction of hydroelastic waves by multiple vertical circular cylinders, Journal of Engineering Mathematics, 113 (2018) 45-64.

[46] K. Ren, G.X. Wu, C.Y. Ji, Wave diffraction and radiation by a vertical circular cylinder standing in a three-dimensional polynya, Journal of Fluids and Structures, 82 (2018) 287-307.

[47] S.P. Timoshenko, K.S. Woinowsky, Theory of plates and shells, McGraw-hill, Singapore, 1959.

[48] J.V. Wehausen, E.V. Laitone, Surface waves, in: Handbuch des Physik, Springer Verlag, Berlin, 1960, pp. 446-778.

[49] M. Abramowitz, I.A. Stegun, Handbook of mathematical functions, Dover press, New York, 1965.

[50] A.J. Burton, G.F. Miller, J.H. Wilkinson, The application of integral equation methods to the numerical solution of some exterior boundary-value problems, Proceedings of the Royal Society of London, A323 (1971) 201-210.

[51] R. Kress, Minimizing the condition number of boundary integral operators in acoustic and electromagnetic scattering The Quarterly Journal of Mechanics and Applied Mathematics, 38 (1985) 323-341.

[52] R.L. Burden, J.D. Faires, Numerical analysis, Brooks Cole, Boston, 2010.

[53] K.R. Drake, R. Eatock Taylor, T. Matsui, Drift of an articulated cylinder in regular waves, Proceedings of the Royal Society of London, A394 (1984) 363-385. 